\title{
Dynamics of a Nonautonomous Stochastic SIS Epidemic Model with Double Epidemic Hypothesis
}

\author{
Haokun Qi, ${ }^{1}$ Lidan Liu, ${ }^{1}$ and Xinzhu Meng ${ }^{1,2}$ \\ ${ }^{1}$ College of Mathematics and Systems Science, Shandong University of Science and Technology, Qingdao 266590, China \\ ${ }^{2}$ State Key Laboratory of Mining Disaster Prevention and Control Co-Founded by Shandong Province and the Ministry of \\ Science and Technology, Shandong University of Science and Technology, Qingdao 266590, China
}

Correspondence should be addressed to Xinzhu Meng; mxz721106@sdust.edu.cn

Received 25 August 2017; Accepted 11 October 2017; Published 8 November 2017

Academic Editor: Benito M. Chen-Charpentier

Copyright (C) 2017 Haokun Qi et al. This is an open access article distributed under the Creative Commons Attribution License, which permits unrestricted use, distribution, and reproduction in any medium, provided the original work is properly cited.

\begin{abstract}
We investigate the dynamics of a nonautonomous stochastic SIS epidemic model with nonlinear incidence rate and double epidemic hypothesis. By constructing suitable stochastic Lyapunov functions and using Has'minskii theory, we prove that there exists at least one nontrivial positive periodic solution of the system. Moreover, the sufficient conditions for extinction of the disease are obtained by using the theory of nonautonomous stochastic differential equations. Finally, numerical simulations are utilized to illustrate our theoretical analysis.
\end{abstract}

\section{Introduction}

The SIS (Susceptible-Infected-Susceptible) model is a basic biological mathematical model describing susceptible and infected epidemic process and is first introduced by Kermack and McKendrick [1]. The SIS model is defined in that individuals start off susceptible, at some stage catch the disease, and after a short infectious period become susceptible again [2]. Therefore, some deterministic SIS epidemic models have been studied by many authors [3-10]. Recently, the authors of [11-13] investigated the epidemic model with double epidemic hypothesis which has two epidemic diseases caused by two different viruses. For example, the deterministic SIS epidemic model with nonlinear saturated incidence rate and double epidemic hypothesis can be expressed as follows [11]:

$$
\begin{aligned}
\dot{S}(t)= & A-d S(t)-\frac{\beta_{1} S(t) I_{1}(t)}{a_{1}+I_{1}(t)}-\frac{\beta_{2} S(t) I_{2}(t)}{a_{2}+I_{2}(t)} \\
& +r_{1} I_{1}(t)+r_{2} I_{2}(t), \\
\dot{I}_{1}(t)= & \frac{\beta_{1} S(t) I_{1}(t)}{a_{1}+I_{1}(t)}-\left(d+\alpha_{1}+r_{1}\right) I_{1}(t)
\end{aligned}
$$

$$
\dot{I}_{2}(t)=\frac{\beta_{2} S(t) I_{2}(t)}{a_{2}+I_{2}(t)}-\left(d+\alpha_{2}+r_{2}\right) I_{2}(t),
$$

where $S(t), I_{1}(t)$, and $I_{2}(t)$ represent the number of susceptibles and infected individuals with viruses $A$ and $B$ at time $t$, respectively. The parameters in model (1) have the following meanings: $A$ is the total input susceptible population size, $d$ represents the natural death rate of $S, I_{1}$, and $I_{2}, \beta_{i}$ represents the disease transmission coefficient between compartments $S$ and $I_{i}(i=1,2), r_{1}$ and $r_{2}$ are the recovery rates of the two diseases, and $\alpha_{1}$ and $\alpha_{2}$ are mortality rates due to diseases, respectively. Functions $\beta_{1} S(t) I_{1}(t) /\left(a_{1}+I_{2}(t)\right)$ and $\beta_{2} S(t) I_{2}(t) /\left(a_{2}+I_{2}(t)\right)$ represent two different types of saturated incidence rates for the two epidemic diseases $I_{1}(t)$ and $I_{2}(t)$. All parameter values are nonnegative.

In the real world, population systems and epidemic systems are inevitably infected by some uncertain environmental disturbances. Hence, many authors have introduced stochastic interferences into differential systems, and the stochastic dynamics of such systems were widely investigated (see [1428]). Moreover, numerous scholars have investigated some stochastic epidemic models (see [29-34]). For example, in [11, 
30] they obtained thresholds of the stochastic system which determines the extinction and persistence of the epidemic. Zhang et al. [29] proved that there is a unique ergodic stationary distribution of his model. We assume that environment fluctuations will manifest themselves mainly as fluctuations in the saturated response rate, so that $\beta_{i} S(t) I_{i}(t) /\left(a_{i}+I_{i}(t)\right) \rightarrow$ $\beta_{i} S(t) I_{i}(t) /\left(a_{i}+I_{i}(t)\right)+\left(\sigma_{i} S(t) I_{i}(t) /\left(a_{i}+I_{i}(t)\right)\right) \dot{B}_{i}(t)(i=1,2)$, where $B(t)=\left(B_{1}(t), B_{2}(t)\right)$ is a standard Brownian motion with intensity $\sigma_{i}>0(i=1,2)$. Therefore, a stochastic model is described by [11]

$$
\begin{aligned}
& \mathrm{d} S(t)=\left(A-d S(t)-\frac{\beta_{1} S(t) I_{1}(t)}{a_{1}+I_{1}(t)}-\frac{\beta_{2} S(t) I_{2}(t)}{a_{2}+I_{2}(t)}\right. \\
& \left.+r_{1} I_{1}(t)+r_{2} I_{2}(t)\right) \mathrm{d} t-\frac{\sigma_{1} S(t) I_{1}(t)}{a_{1}+I_{1}(t)} \mathrm{d} B_{1}(t) \\
& -\frac{\sigma_{2} S(t) I_{2}(t)}{a_{2}+I_{2}(t)} \mathrm{d} B_{2}(t), \\
& \mathrm{d} I_{1}(t)=\left(\frac{\beta_{1} S(t) I_{1}(t)}{a_{1}+I_{1}(t)}-\left(d+\alpha_{1}+r_{1}\right) I_{1}(t)\right) \mathrm{d} t \\
& +\frac{\sigma_{1} S(t) I_{1}(t)}{a_{1}+I_{1}(t)} \mathrm{d} B_{1}(t), \\
& \mathrm{d} I_{2}(t)=\left(\frac{\beta_{2} S(t) I_{2}(t)}{a_{2}+I_{2}(t)}-\left(d+\alpha_{2}+r_{2}\right) I_{2}(t)\right) \mathrm{d} t \\
& +\frac{\sigma_{2} S(t) I_{2}(t)}{a_{2}+I_{2}(t)} \mathrm{d} B_{2}(t) .
\end{aligned}
$$

However, many infectious diseases of human fluctuate over time and often show the seasonal morbidity. Therefore, the existence of periodic solutions of some nonautonomous epidemic models was explored [35-37]. Recently, many scholars focused on nonautonomous stochastic periodic systems. With the development of stochastic differential equations and application of Has'minskii theory, the existence of stochastic periodic solution has been studied [23, 38, 39]. In [23], Zhang et al. considered a nonautonomous stochastic Lotka-Volterra predator-prey model with impulsive effects; they got thresholds for stochastic persistence and extinction of the system. Authors of [38-40] investigated periodic solution of a stochastic nonautonomous epidemic model.

Based on the discussion above, in this paper, we consider a nonautonomous stochastic SIS model with periodic coefficients

$$
\begin{gathered}
\mathrm{d} S(t)=\left(A(t)-d(t) S(t)-\frac{\beta_{1}(t) S(t) I_{1}(t)}{a_{1}(t)+I_{1}(t)}\right. \\
\left.-\frac{\beta_{2}(t) S(t) I_{2}(t)}{a_{2}(t)+I_{2}(t)}+r_{1}(t) I_{1}(t)+r_{2}(t) I_{2}(t)\right) \mathrm{d} t \\
-\frac{\sigma_{1}(t) S(t) I_{1}(t)}{a_{1}(t)+I_{1}(t)} \mathrm{d} B_{1}(t)-\frac{\sigma_{2}(t) S(t) I_{2}(t)}{a_{2}(t)+I_{2}(t)} \mathrm{d} B_{2}(t),
\end{gathered}
$$

$$
\begin{aligned}
& \mathrm{d} I_{1}(t)=\left(\frac{\beta_{1}(t) S(t) I_{1}(t)}{a_{1}(t)+I_{1}(t)}\right. \\
&-\left.\left(d(t)+\alpha_{1}(t)+r_{1}(t)\right) I_{1}(t)\right) \mathrm{d} t \\
&+ \frac{\sigma_{1}(t) S(t) I_{1}(t)}{a_{1}(t)+I_{1}(t)} \mathrm{d} B_{1}(t), \\
& \mathrm{d} I_{2}(t)=\left(\frac{\beta_{2}(t) S(t) I_{2}(t)}{a_{2}(t)+I_{2}(t)}\right. \\
&\left.-\left(d(t)+\alpha_{2}(t)+r_{2}(t)\right) I_{2}(t)\right) \mathrm{d} t \\
&+\frac{\sigma_{2}(t) S(t) I_{2}(t)}{a_{2}(t)+I_{2}(t)} \mathrm{d} B_{2}(t),
\end{aligned}
$$

where the parameter functions $A(t), d(t), \beta_{i}(t), a_{i}(t), r_{i}(t)$, $\alpha_{i}(t), \sigma_{i}(t)(i=1,2)$ are positive, nonconstant, and continuous periodic functions with positive period $T$.

To the best of our knowledge, there are only few works on research of nonautonomous stochastic epidemic models with nonlinear saturated incidence rate and double epidemic hypothesis. Therefore, based on an autonomous stochastic epidemic model, we propose a nonautonomous stochastic model and investigate the existence of stochastic periodic solution and the extinction of the two epidemic diseases.

This paper is organized as follows. In Section 2, we give some definitions and known results. In Section 3, we prove that system (3) has a unique global positive solution. In Section 4, we present sufficient condition for the existence a nontrivial positive periodic solution of system (3). In Section 5, we obtain the sufficient conditions of system (3) for extinction of the two epidemic diseases. In Section 6, we carry out a series of numerical simulations to illustrate our theoretical findings.

\section{Preliminaries}

Throughout this paper, let $(\Omega, \mathscr{F}, \mathbb{P})$ be a complete probability space with a filtration $\left\{\mathscr{F}_{t}\right\}_{t \geq 0}$ satisfying the usual conditions (i.e., it is increasing and right continuous while $\mathscr{F}_{0}$ contains all $\mathbb{P}$-null sets). The function $B_{i}(t)(i=1,2,3,4)$ is defined on this complete probability space.

For simplicity, some notations are given first. If $f(t)$ is an integrable function defined on $[0, \infty)$, define $\langle f\rangle_{t}=$ $(1 / t) \int_{0}^{t} f(s) d s, t>0$. If $f(t)$ is a bounded function on $[0, \infty)$, define $f^{l}=\inf _{t \in[0, \infty)} f(t)$ and $f^{u}=\sup _{t \in[0, \infty)} f(t)$.

Here we present some basic theory in stochastic differential equations which are introduced in [41].

In general, consider the $l$-dimensional stochastic differential equation

$$
\mathrm{d} X(t)=f(X(t), t) \mathrm{d} t+g(X(t), t) d B(t), \quad t \geq t_{0},
$$

with initial value $x\left(t_{0}\right)=x_{0} \in \mathbb{R}^{l} . B(t)$ stands for a $l$-dimensional standard Brownian motion defined on the 
complete probability space $\left(\Omega, \mathscr{F},\left\{\mathscr{F}_{t}\right\}_{t \geq 0}, \mathbb{P}\right)$. Denote by $C^{2,1}\left(\mathbb{R}^{l} \times\left[t_{0}, \infty\right] ; \mathbb{R}_{+}\right)$the family of all nonnegative functions $V(X, t)$ defined on $\mathbb{R}^{l} \times\left[t_{0}, \infty\right]$ such that they are continuously twice differentiable in $X$ and once in $t$. The differential operator $L$ of (4) is defined by [41]

$$
\begin{aligned}
L= & \frac{\partial}{\partial t}+\sum f_{i}(X, t) \frac{\partial}{\partial X_{i}} \\
& +\frac{1}{2} \sum_{i, j=1}^{l}\left[g^{T}(X, t) g(X, t)\right]_{i j} \frac{\partial^{2}}{\partial X_{i} \partial X_{j}} .
\end{aligned}
$$

If $L$ acts on a function $V \in C^{2,1}\left(\mathbb{R}^{l} \times\left[t_{0}, \infty\right] ; \mathbb{R}_{+}\right)$, then

$$
\begin{aligned}
L V(X, t)= & V_{t}(X, t)+V_{X}(X, t) f(X, t) \\
& +\frac{1}{2} \operatorname{trace}\left[g^{T}(X, t) V_{X X}(X, t) g(X, t)\right],
\end{aligned}
$$

where $V_{t}=\partial V / \partial t, V_{X}=\left(\partial V / \partial x_{1}, \ldots, \partial V / \partial x_{l}\right)$, and $V_{X X}=$ $\left(\partial^{2} V / \partial X_{i} \partial X_{j}\right)_{l \times l}$. In view of Itô's formula, if $X(t) \in \mathbb{R}^{l}$, then

$$
\begin{aligned}
\mathrm{d} V(X(t), t)= & L V(x(t), t) \mathrm{d} t \\
& +V_{X}(X(t), t) g(X(t), t) d B(t) .
\end{aligned}
$$

Definition 1 (see [42]). A stochastic process $\xi(t)=$ $\xi(t, \omega)(-\infty<t<+\infty)$ is said to be periodic with period $T$ if for every finite sequence of numbers $t_{1}, t_{2}, \ldots, t_{n}$ the joint distribution of random variables $\xi\left(t_{1}+h\right), \xi\left(t_{2}+h\right), \ldots, \xi\left(t_{n}+\right.$ $h$ ) is independent of $h$, where $h=k T, k= \pm 1, \pm 2, \ldots$.

It is shown in [42] that a Markov process $x(t)$ is $T$ periodic if and only if its transition probability function is $T$ periodic and the function $\mathbb{P}_{0}(t, A)=\mathbb{P}\{x(t) \in A\}$ satisfies the equation

$$
\begin{aligned}
\mathbb{P}_{0}(s, A) & =\int_{\mathbb{R}^{l}} \mathbb{P}_{0}(s, d x) \mathbb{P}(s, x, s+T, A) \\
& =\mathbb{P}_{0}(s+T, A) .
\end{aligned}
$$

Consider the following equation:

$$
\begin{aligned}
X(t)= & X\left(t_{0}\right)+\int_{t_{0}}^{t} b(s, X(s)) d s \\
& +\sum_{r=1}^{k} \int_{t_{0}}^{t} \sigma_{r}(s, X(s)) d B_{r}(s), \quad X \in \mathbb{R}^{l} .
\end{aligned}
$$

Lemma 2 (see [42]). Suppose that coefficients of (9) are Tperiodic in $t$ and satisfy the condition

$$
\begin{aligned}
& |b(s, x)-b(s, y)|+\sum_{r=1}^{k}\left|\sigma_{r}(s, x)-\sigma_{r}(s, y)\right| \\
& \quad \leq P|x-y| \\
& |b(s, x)|+\sum_{r=1}^{k}\left|\sigma_{r}(s, x)\right| \leq P(1+|x|),
\end{aligned}
$$

in every cylinder $I \times U$, where $P$ is a constant. And suppose further that there exists a function $V(t, x) \in C^{2}$ in $\mathbb{R}^{d}$ which is $T$-periodic in $t$ and satisfies the following conditions:

$$
\left(A_{1}\right) \inf _{|x| \geq \mathbb{R}} V(t, x) \rightarrow \infty \text { as } \mathbb{R} \rightarrow \infty \text {. }
$$

$\left(A_{2}\right) L V(t, x) \leq-1$ outside some compact set, where the operator $L$ is given by

$$
\begin{aligned}
L & =\frac{\partial}{\partial t}+\sum_{i=1}^{l} b_{i}(t, x) \frac{\partial}{\partial x_{i}}+\frac{1}{2} \sum_{i, j=1}^{l} a_{i j}(t, x) \frac{\partial^{2}}{\partial x_{i} \partial x_{j}}, \\
a_{i j} & =\sum_{r=1}^{k} \sigma_{r}^{i}(t, x) \sigma_{r}^{j}(t, x) .
\end{aligned}
$$

Then there exists a solution of (9) which is a T-periodic Markov process.

Lemma 3 (see [2], strong law of large numbers). Let $M=$ $\left\{M_{t}\right\}_{t} \geq 0$ be a real-valued continuous local martingale vanishing at $t=0$.

Then

$$
\begin{aligned}
& \lim _{t \rightarrow \infty}\langle M, M\rangle_{t}=\infty \quad \text { a.s. } \Longrightarrow \\
& \lim _{t \rightarrow \infty} \frac{M_{t}}{\langle M, M\rangle_{t}}=0 \quad \text { a.s. }
\end{aligned}
$$

and also

$$
\begin{aligned}
\limsup _{t \rightarrow \infty} \frac{\langle M, M\rangle_{t}}{t}<\infty & \text { a.s. } \Longrightarrow \\
\lim _{t \rightarrow \infty} \frac{M_{t}}{t} & =0 \quad \text { a.s. }
\end{aligned}
$$

\section{Existence and Uniqueness of the Global Positive Solution}

In this section, we prove that system (3) has a unique global positive solution.

Theorem 4. For any initial value $\left(S(0), I_{1}(0), I_{2}(0)\right) \in R_{+}^{3}$, there is a unique positive solution $\left(S(t), I_{1}(t), I_{2}(t)\right)$ of (3) on $t \geq 0$ and the solution will remain in $R_{+}^{3}$ with probability one.

Proof. From system (3), we can get

$$
\begin{aligned}
\frac{\mathrm{d}\left(S(t)+I_{1}(t)+I_{2}(t)\right)}{\mathrm{d} t} \\
=A(t)-d(t)\left(S(t)+I_{1}(t)+I_{2}(t)\right) \\
\quad-\left(\alpha_{1}(t) I_{1}(t)+\alpha_{2}(t) I_{2}(t)\right) \\
\leq A(t)-d(t)\left(S(t)+I_{1}(t)+I_{2}(t)\right) \\
\leq A^{u}-d^{l}\left(S(t)+I_{1}(t)+I_{2}(t)\right) .
\end{aligned}
$$

Then

$$
\lim _{t \rightarrow+\infty}\left(S(t)+I_{1}(t)+I_{2}(t)\right) \leq \frac{A^{u}}{d^{l}}
$$


obviously, we have

$$
\begin{gathered}
\limsup _{t \rightarrow+\infty} S(t) \leq \frac{A^{u}}{d^{l}}, \\
\limsup _{t \rightarrow+\infty} I_{1}(t) \leq \frac{A^{u}}{d^{l}}, \\
\limsup _{t \rightarrow+\infty} I_{2}(t) \leq \frac{A^{u}}{d^{l}} .
\end{gathered}
$$

Since the coefficients of system (3) satisfy the local Lipschitz conditions, then for any given initial value $\left(S(0), I_{1}(0), I_{2}(0)\right) \in R_{+}^{3}$, there is a unique local solution $\left(S(t), I_{1}(t), I_{2}(t)\right)$ on $t \in\left[0, \tau_{e}\right)$, where $\tau_{e}$ is the explosion time. To demonstrate that this solution is global, we only need to prove that $\tau_{e}=\infty$ a.s.

Let $k_{0}>0$ be sufficiently large for any initial value $S(0), I_{1}(0)$, and $I_{2}(0)$ lying within the interval $\left[1 / k_{0}, k\right]$. For each integer $k \geq k_{0}$, define the following stopping time:

$$
\begin{aligned}
\tau_{k} & =\inf \left\{t \in\left[0, \tau_{e}\right): \min \left\{S(t), I_{1}(t), I_{2}(t)\right\}\right. \\
& \left.\leq \frac{1}{k} \text { or } \max \left\{S(t), I_{1}(t), I_{2}(t)\right\} \geq k\right\},
\end{aligned}
$$

where we set $\inf \emptyset=\infty$ (as usual $\emptyset$ denotes the empty set). Clearly, $\tau_{k}$ is increasing as $k \rightarrow \infty$. Let $\tau_{\infty}=\lim _{k \rightarrow \infty} \tau_{k}$; hence $\tau_{\infty} \leq \tau_{k}$ a.s. Next, we only need to verify $\tau_{\infty}=\infty$ a.s. If this statement is false, then there exist two constants $T>0$ and $\epsilon \in(0,1)$ such that

$$
\mathbb{P}\left\{\tau_{\infty} \leq T\right\}>\epsilon
$$

Thus there is an integer $k_{1} \geq k_{0}$ such that

$$
\mathbb{P}\left\{\tau_{k} \leq T\right\} \geq \epsilon, \quad k \geq k_{1} .
$$

Define a $C^{2}$-function $V: \mathbb{R}_{+}^{3} \rightarrow \mathbb{R}_{+}$as follows:

$$
\begin{aligned}
V\left(S, I_{1}, I_{2}\right)= & S-1-\ln S+I_{1}-1-\ln I_{1}+I_{2}-1 \\
& -\ln I_{2} ;
\end{aligned}
$$

the nonnegativity of this function can be obtained from

$$
u-1-\ln u \geq 0, \quad u>0 .
$$

Applying Itô's formula yields

$$
\begin{aligned}
\mathrm{d} V\left(S, I_{1}, I_{2}\right)= & L V\left(S, I_{1}, I_{2}\right) \mathrm{d} t \\
& +\left(2 S I_{1}-S-I_{1}\right) \frac{\sigma_{1}(t)}{a_{1}(t)+I_{1}} d B_{1}(t) \\
& +\left(2 S I_{2}-S-I_{2}\right) \frac{\sigma_{2}(t)}{a_{2}(t)+I_{2}} d B_{2}(t),
\end{aligned}
$$

where

$$
\begin{aligned}
L V & =\left(1-\frac{1}{S}\right)\left(A(t)-d(t) S-\frac{\beta_{1}(t) S I_{1}}{a_{1}(t)+I_{1}}\right. \\
& \left.-\frac{\beta_{2}(t) S I_{2}}{a_{2}(t)+I_{2}}+r_{1}(t) I_{1}+r_{2}(t) I_{2}\right)+\frac{1}{2}
\end{aligned}
$$

$$
\begin{aligned}
& \frac{\sigma_{1}^{2}(t) I_{1}^{2}}{\left(a_{1}(t)+I_{1}\right)^{2}}+\frac{1}{2} \frac{\sigma_{2}^{2}(t) I_{2}^{2}}{\left(a_{2}(t)+I_{2}\right)^{2}}+\left(1-\frac{1}{I_{1}}\right) \\
\cdot & \left(\frac{\beta_{1}(t) S I_{1}}{a_{1}(t)+I_{1}}-\left(d(t)+\alpha_{1}(t)+r_{1}(t)\right) I_{1}\right)+\frac{1}{2} \\
\cdot & \frac{\sigma_{1}^{2}(t) S^{2}}{\left(a_{1}(t)+I_{1}\right)^{2}}+\left(1-\frac{1}{I_{2}}\right)\left(\frac{\beta_{2}(t) S I_{2}}{a_{2}(t)+I_{2}}\right. \\
- & \left.\left(d(t)+\alpha_{2}(t)+r_{2}(t)\right) I_{2}\right)+\frac{1}{2} \frac{\sigma_{2}^{2}(t) S^{2}}{\left(a_{2}(t)+I_{2}\right)^{2}} \\
= & A(t)-d(t) S-\frac{A(t)}{S}+d(t)+\frac{\beta_{1}(t) I_{1}}{a_{1}(t)+I_{1}} \\
+ & \frac{\beta_{2}(t) I_{2}}{a_{2}(t)+I_{2}}-\frac{r_{1}(t) I_{1}}{S}-\frac{r_{2}(t) I_{2}}{S} \\
+ & \frac{\sigma_{1}^{2}(t) I_{1}^{2}}{2\left(a_{1}(t)+I_{1}\right)^{2}}+\frac{\sigma_{2}^{2}(t) I_{2}^{2}}{2\left(a_{2}(t)+I_{2}\right)^{2}}-(d(t) \\
+ & \frac{\sigma_{1}^{2 u} A^{2 u}}{2 a_{1}^{2 l} d^{2 l}}+\frac{\sigma_{2}^{2 u} A^{2 u}}{2 a_{2}^{2 l} d^{2 l}}:=K, \\
+ & \alpha(t)+\alpha_{1}(t)+r_{2}(t)+\frac{\sigma_{2}^{2}(t) S_{1}^{2}}{2\left(a_{2}(t)+I_{2}\right)^{2}} \leq A^{u} \\
+ & \frac{\sigma_{1}^{2}(t) S_{1}^{2}(t)+I_{1}}{2\left(a_{1}(t)+I_{1}\right)^{2}}-\left(d(t)+\alpha_{1}(t)+r_{1}(t)\right. \\
\left.a_{1}(t)+\alpha_{2}(t)\right) I_{2}-\frac{\beta_{2}(t) S}{a_{2}(t)+I_{2}} & \sigma_{2}^{2 u} \\
+ & \frac{\sigma_{1}^{u}}{2}+\beta_{2}^{u} \\
+ &
\end{aligned}
$$

where $K$ is a positive constant.

$$
\text { So we have }
$$

$$
\begin{aligned}
\mathrm{d} V\left(S, I_{1}, I_{2}\right) \leq & K \mathrm{~d} t \\
& +\left(2 S I_{1}-S-I_{1}\right) \frac{\sigma_{1}(t)}{a_{1}(t)+I_{1}} d B_{1}(t) \\
& +\left(2 S I_{2}-S-I_{2}\right) \frac{\sigma_{2}(t)}{a_{2}(t)+I_{2}} d B_{2}(t) .
\end{aligned}
$$

Integrating (24) from 0 to $\tau_{k} \wedge T$ and taking expectations on both sides yield

$$
\begin{gathered}
\mathbb{E} V\left(S\left(\tau_{k} \wedge T\right), I_{1}\left(\tau_{k} \wedge T\right), I_{2}\left(\tau_{k} \wedge T\right)\right) \\
\leq V\left(S(0), I_{1}(0), I_{2}(0)\right)+K T .
\end{gathered}
$$

Let $\Omega_{k}=\left\{\tau_{k} \leq T\right\}$; from inequality (25) we can see that $P\left(\Omega_{k}\right) \geq \epsilon$. We have

$$
\begin{gathered}
V\left(S\left(\tau_{k} \wedge T\right), I_{1}\left(\tau_{k} \wedge T\right), I_{2}\left(\tau_{k} \wedge T\right)\right) \\
\quad \geq(k-1-\ln k) \wedge\left(\frac{1}{k}-1-\ln \frac{1}{k}\right) .
\end{gathered}
$$


By (25) and (26), one has

$$
\begin{aligned}
& V\left(S(0), I_{1}(0), I_{2}(0)\right)+K T \geq \mathbb{E}\left[1 \Omega_{k}(\omega)\right. \\
& \left.\quad \cdot V\left(S\left(\tau_{k} \wedge T\right), I_{1}\left(\tau_{k} \wedge T\right), I_{2}\left(\tau_{k} \wedge T\right)\right)\right] \geq \epsilon(k \\
& \quad-1-\ln k) \wedge\left(\frac{1}{k}-1-\ln \frac{1}{k}\right),
\end{aligned}
$$

where $1 \Omega_{k}$ is the indicator function of $\Omega_{k}$.
Let $k \rightarrow \infty$; we have

$$
\infty>V\left(S(0), I_{1}(0), I_{2}(0)\right)+K T=\infty .
$$

So we obtain $\tau_{\infty}=\infty$. The proof is completed.

\section{Existence of Nontrivial $T$-Periodic Solution}

In this section, we verify that system (3) admits at least one nontrivial positive $T$-periodic solution. Define

$$
\Re=\sum_{i=1}^{2} \frac{\left\langle A \alpha_{i} \beta_{i}\right\rangle_{T}}{\left\langle d+\alpha_{i}+r_{i}+\sigma_{i}^{2} A^{2 u} / 2 a_{i}^{2} d^{2 l}\right\rangle_{T}\left\langle d+\sigma_{1}^{2} / 2+\sigma_{2}^{2} / 2\right\rangle_{T}\left\langle A+a_{1} \alpha_{1}+a_{2} \alpha_{2}\right\rangle_{T}} .
$$

Theorem 5. When $\left(a_{1}(t) r_{1}(t) /\left(\beta_{1}(t)-a_{1}(t) d(t)\right)\right)^{l}>A^{u} / d^{l}$ and $\left(a_{2}(t) r_{2}(t) /\left(\beta_{2}(t)-a_{2}(t) d(t)\right)\right)^{l}>A^{u} / d^{l}$ hold, if $\mathfrak{R}>1$, then there exists a nontrivial positive T-periodic solution of system (3).

Proof. Define a $C^{2}$-function $\bar{V}:[0, \infty) \times \mathbb{R}_{+}^{3} \rightarrow \mathbb{R}_{+}$:

$$
\begin{aligned}
& \bar{V}\left(t, S, I_{1}, I_{2}\right)=M\left(-\left(e_{1}+e_{2}\right) \ln S-c_{1} \ln I_{1}-c_{2} \ln I_{2}\right. \\
& \left.+S+I_{1}+I_{2}+\omega(t)\right)-\ln S-I_{1}-I_{2}+\frac{1}{\theta+1}(S \\
& \left.\quad+I_{1}+I_{2}\right)^{\theta+1}=M\left(V_{1}+\omega(t)\right)+V_{2}+V_{3}+V_{4} \\
& \quad+V_{5},
\end{aligned}
$$

where

$$
\begin{aligned}
& c_{1} \\
& =\frac{\left\langle A \alpha_{1} \beta_{1}\right\rangle_{T}}{\left\langle d+\alpha_{1}+r_{1}+\sigma_{1}^{2} A^{2 u} / 2 a_{1}^{2} d^{2 l}\right\rangle_{T}^{2}\left\langle d+\sigma_{1}^{2} / 2+\sigma_{2}^{2} / 2\right\rangle_{T}}, \\
& e_{1} \\
& =\frac{\left\langle A \alpha_{1} \beta_{1}\right\rangle_{T}}{\left\langle d+\alpha_{1}+r_{1}+\sigma_{1}^{2} A^{2 u} / 2 a_{1}^{2} d^{2 l}\right\rangle_{T}\left\langle d+\sigma_{1}^{2} / 2+\sigma_{2}^{2} / 2\right\rangle_{T}^{2}}, \\
& c_{2} \\
& =\frac{\left\langle A \alpha_{2} \beta_{2}\right\rangle_{T}}{\left\langle d+\alpha_{2}+r_{2}+\sigma_{2}^{2} A^{2 u} / 2 a_{2}^{2} d^{2 l}\right\rangle_{T}^{2}\left\langle d+\sigma_{1}^{2} / 2+\sigma_{2}^{2} / 2\right\rangle_{T}}, \\
& e_{2} \\
& =\frac{\left\langle A \alpha_{2} \beta_{2}\right\rangle_{T}}{\left\langle d+\alpha_{2}+r_{2}+\sigma_{2}^{2} A^{2 u} / 2 a_{2}^{2} d^{2 l}\right\rangle_{T}\left\langle d+\sigma_{1}^{2} / 2+\sigma_{2}^{2} / 2\right\rangle_{T}^{2}},
\end{aligned}
$$

and $0<\theta<\min \left\{1, d^{l} /\left(\sigma_{1}^{2 u}+\sigma_{2}^{2 u}\right)\right\} ; M$ is a sufficiently large positive constant and satisfies the following conditions:

$$
\begin{gathered}
d^{l}-\theta\left(\sigma_{1}^{2 u}+\sigma_{2}^{2 u}\right)>0, \\
-M \lambda+\max \{D, E\} \leq-2,
\end{gathered}
$$

where

$$
\begin{aligned}
\lambda= & \left\langle A+a_{1} \alpha_{1}+a_{2} \alpha_{2}\right\rangle_{T}(\Re-1), \\
D= & \sup _{\left(S, I_{1}, I_{2}\right) \in R_{+}^{3}}\left\{\left(d^{u}+\alpha_{2}^{u}+r_{2}^{u}\right) I_{2}\right. \\
& -\frac{1}{2}\left(d^{l}-\theta\left(\sigma_{1}^{2 u}+\sigma_{2}^{2 u}\right)\right) I_{2}^{\theta+1}+O+d^{u}+\beta_{1}^{u}+\beta_{2}^{u} \\
& \left.+\frac{\sigma_{1}^{2 u}}{2}+\frac{\sigma_{2}^{2 u}}{2}\right\}, \\
E= & \sup _{\left(S, I_{1}, I_{2}\right) \in R_{+}^{3}}\left\{\left(d^{u}+\alpha_{1}^{u}+r_{1}^{u}\right) I_{1}\right. \\
& -\frac{1}{2}\left(d^{l}-\theta\left(\sigma_{1}^{2 u}+\sigma_{2}^{2 u}\right)\right) I_{1}^{\theta+1}+O+d^{u}+\beta_{1}^{u}+\beta_{2}^{u} \\
& \left.+\frac{\sigma_{1}^{2 u}}{2}+\frac{\sigma_{2}^{2 u}}{2}\right\},
\end{aligned}
$$

where

$$
\begin{aligned}
O= & \sup _{\left(S, I_{1}, I_{2}\right) \in \mathbb{R}_{+}^{3}}\left\{A^{u}\left(S+I_{1}+I_{2}\right)^{\theta}\right. \\
& \left.-\frac{1}{2}\left(d^{l}-\theta\left(\sigma_{1}^{2 u}+\sigma_{2}^{2 u}\right)\right)\left(S+I_{1}+I_{2}\right)^{\theta+1}\right\}<\infty .
\end{aligned}
$$

Next we prove that condition $\left(A_{1}\right)$ in Lemma 2 holds. It is easy to check that $\bar{V}\left(t, S, I_{1}, I_{2}\right)$ is a $T$-periodic function in $t$ and satisfies

$$
\liminf _{K \rightarrow \infty,\left(S, I_{1}, I_{2}\right) \in \mathbb{R}_{+}^{3} \backslash \mathbb{U}_{k}} \bar{V}\left(t, S, I_{1}, I_{2}\right)=\infty,
$$

where $\mathbb{U}_{k}=(1 / k, k) \times(1 / k, k) \times(1 / k, k)$ and $k>1$ is a sufficiently large number. 
By Itô's formula, we obtain

$$
\begin{aligned}
& L V_{1}=-\frac{e_{1}+e_{2}}{S}\left(A(t)-d(t) S-\frac{\beta_{1}(t) S I_{1}}{a_{1}(t)+I_{1}}\right. \\
& \left.-\frac{\beta_{2}(t) S I_{2}}{a_{2}(t)+I_{2}}+r_{1}(t) I_{1}+r_{2}(t) I_{2}\right) \\
& -\frac{c_{1}}{I_{1}}\left(\frac{\beta_{1}(t) S I_{1}}{a_{1}(t)+I_{1}}-\left(d(t)+\alpha_{1}(t)+r_{1}(t)\right) I_{1}\right) \\
& +\frac{e_{1}+e_{2}}{2} \frac{\sigma_{1}^{2}(t) I_{1}^{2}}{\left(a_{1}(t)+I_{1}\right)^{2}}-\frac{c_{2}}{I_{2}}\left(\frac{\beta_{2}(t) S I_{2}}{a_{2}(t)+I_{2}}\right. \\
& \left.-\left(d(t)+\alpha_{2}(t)+r_{2}(t)\right) I_{2}\right)+\frac{e_{1}+e_{2}}{2} \\
& \cdot \frac{\sigma_{2}^{2}(t) I_{2}^{2}}{\left(a_{2}(t)+I_{2}\right)^{2}}+\frac{c_{1}}{2} \frac{\sigma_{1}^{2}(t) S^{2}}{\left(a_{1}(t)+I_{1}\right)^{2}}+\frac{c_{2}}{2} \\
& \cdot \frac{\sigma_{2}^{2}(t) S^{2}}{\left(a_{2}(t)+I_{2}\right)^{2}}+A(t)-d(t)\left(S+I_{1}+I_{2}\right)-\alpha_{1}(t) \\
& \cdot I_{1}-\alpha_{2}(t) I_{2} \leq-e_{1} \frac{A(t)}{S}-\frac{c_{1} \beta_{1}(t) S}{a_{1}(t)+I_{1}}-\alpha_{1}(t) \\
& \cdot\left(a_{1}(t)+I_{1}\right)-e_{2} \frac{A(t)}{S}-\frac{c_{2} \beta_{2}(t) S}{a_{2}(t)+I_{2}}-\alpha_{2}(t) \\
& \cdot\left(a_{2}(t)+I_{2}\right)+c_{1}\left(d(t)+\alpha_{1}(t)+r_{1}(t)\right. \\
& \left.+\frac{\sigma_{1}^{2}(t) A^{2 u}}{2 a_{1}^{2}(t) d^{2 l}}\right)+e_{1}\left(d(t)+\frac{\sigma_{1}^{2}(t)}{2}+\frac{\sigma_{2}^{2}(t)}{2}\right) \\
& +c_{2}\left(d(t)+\alpha_{2}(t)+r_{2}(t)+\frac{\sigma_{2}^{2}(t) A^{2 u}}{2 a_{2}^{2}(t) d^{2 l}}\right) \\
& +e_{2}\left(d(t)+\frac{\sigma_{1}^{2}(t)}{2}+\frac{\sigma_{2}^{2}(t)}{2}\right)-\left(e_{1}+e_{2}\right)\left(\frac{r_{1}(t)}{S}\right. \\
& \left.+d(t)-\frac{\beta_{1}(t)}{a_{1}(t)}\right) I_{1}-\left(e_{1}+e_{2}\right)\left(\frac{r_{2}(t)}{S}+d(t)\right. \\
& \left.-\frac{\beta_{2}(t)}{a_{2}(t)}\right) I_{2}+A(t)+a_{1}(t) \alpha_{1}(t)+a_{2}(t) \alpha_{2}(t) \\
& \leq-3\left(A(t) \alpha_{1}(t) \beta_{1}(t) c_{1} e_{1}\right)^{1 / 3} \\
& -3\left(A(t) \alpha_{2}(t) \beta_{2}(t) c_{2} e_{2}\right)^{1 / 3}+e_{1}\left(d(t)+\frac{\sigma_{1}^{2}(t)}{2}\right. \\
& \left.+\frac{\sigma_{2}^{2}(t)}{2}\right)+c_{1}\left(d(t)+\alpha_{1}(t)+r_{1}(t)\right. \\
& \left.+\frac{\sigma_{1}^{2}(t) A^{2 u}}{2 a_{1}^{2}(t) d^{2 l}}\right)+c_{2}\left(d(t)+\alpha_{2}(t)+r_{2}(t)\right. \\
& \left.+\frac{\sigma_{2}^{2}(t) A^{2 u}}{2 a_{2}^{2}(t) d^{2 l}}\right)+e_{2}\left(d(t)+\frac{\sigma_{1}^{2}(t)}{2}+\frac{\sigma_{2}^{2}(t)}{2}\right)
\end{aligned}
$$

$$
\begin{aligned}
& +A(t)+a_{1}(t) \alpha_{1}(t)+a_{2}(t) \alpha_{2}(t)-\left(e_{1}+e_{2}\right) \\
& \cdot\left(\frac{r_{1}(t)}{S}+d(t)-\frac{\beta_{1}(t)}{a_{1}(t)}\right) I_{1}-\left(e_{1}+e_{2}\right)\left(\frac{r_{2}(t)}{S}\right. \\
& \left.+d(t)-\frac{\beta_{2}(t)}{a_{2}(t)}\right) I_{2}:=\mathfrak{R}_{0}(t)-\left(e_{1}+e_{2}\right)\left(\frac{r_{1}(t)}{S}\right. \\
& \left.+d(t)-\frac{\beta_{1}(t)}{a_{1}(t)}\right) I_{1}-\left(e_{1}+e_{2}\right)\left(\frac{r_{2}(t)}{S}+d(t)\right. \\
& \left.-\frac{\beta_{2}(t)}{a_{2}(t)}\right) I_{2},
\end{aligned}
$$

where

$$
\begin{aligned}
\mathfrak{R}_{0}(t)= & 3\left(A(t) \alpha_{1}(t) \beta_{1}(t) c_{1} e_{1}\right)^{1 / 3} \\
& +c_{1}\left(d(t)+\alpha_{1}(t)+r_{1}(t)+\frac{\sigma_{1}^{2}(t) A^{2 u}}{2 a_{1}^{2}(t) d^{2 l}}\right) \\
& +e_{1}\left(d(t)+\frac{\sigma_{1}^{2}(t)}{2}+\frac{\sigma_{2}^{2}(t)}{2}\right) \\
& -3\left(A(t) \alpha_{2}(t) \beta_{2}(t) c_{2} e_{2}\right)^{1 / 3} \\
& +c_{2}\left(d(t)+\alpha_{2}(t)+r_{2}(t)+\frac{\sigma_{2}^{2}(t) A^{2 u}}{2 a_{2}^{2}(t) d^{2 l}}\right) \\
& +e_{2}\left(d(t)+\frac{\sigma_{1}^{2}(t)}{2}+\frac{\sigma_{2}^{2}(t)}{2}\right)+A(t) \\
& +a_{1}(t) \alpha_{1}(t)+a_{2}(t) \alpha_{2}(t) .
\end{aligned}
$$

Note that $\left(a_{1}(t) r_{1}(t) /\left(\beta_{1}(t)-a_{1}(t) d(t)\right)\right)^{l}>A^{u} / d^{l}$ and $\left(a_{2}(t) r_{2}(t) /\left(\beta_{2}(t)-a_{2}(t) d(t)\right)\right)^{l}>A^{u} / d^{l}$ hold; then

$$
L\left(V_{1}\right) \leq \mathfrak{R}_{0}(t)
$$

Define the $T$-periodic function $\omega(t)$ satisfying

$$
\omega^{\prime}(t)=\left\langle\mathfrak{R}_{0}\right\rangle_{T}-\mathfrak{R}_{0}(t)
$$

So

$$
\begin{aligned}
L\left(V_{1}+\omega(t)\right) & \leq\left\langle\mathfrak{R}_{0}\right\rangle_{T} \\
& =-\left\langle A+a_{1} \alpha_{1}+a_{2} \alpha_{2}\right\rangle_{T}(\mathfrak{R}-1) \\
& :=-\lambda .
\end{aligned}
$$

Applying Itô's formula, we can also have 


$$
\begin{aligned}
& L V_{2}=-\frac{A(t)}{S}+d(t)+\frac{\beta_{1}(t) I_{1}}{a_{1}(t)+I_{1}}+\frac{\beta_{2}(t) I_{2}}{a_{2}(t)+I_{2}} \\
& -\frac{r_{1}(t) I_{1}}{S}-\frac{r_{2}(t) I_{2}}{S}+\frac{\sigma_{1}^{2}(t) I_{1}^{2}}{2\left(a_{1}(t)+I_{1}\right)^{2}} \\
& +\frac{\sigma_{2}^{2}(t) I_{2}^{2}}{2\left(a_{2}(t)+I_{2}\right)^{2}} \leq-\frac{A^{l}}{S}+d^{u}+\beta_{1}^{u}+\beta_{2}^{u}+\frac{\sigma_{1}^{2 u}}{2} \\
& +\frac{\sigma_{2}^{2 u}}{2} \\
& L V_{3}=-\frac{\beta_{1}(t) S I_{1}}{a_{1}(t)+I_{1}}+\left(d(t)+\alpha_{1}(t)+r_{1}(t)\right) I_{1} \leq\left(d^{u}\right. \\
& \left.+\alpha_{1}^{u}+r_{1}^{u}\right) I_{1} \text {, } \\
& L V_{4}=-\frac{\beta_{2}(t) S I_{2}}{a_{2}(t)+I_{2}}+\left(d(t)+\alpha_{2}(t)+r_{2}(t)\right) I_{2} \leq\left(d^{u}\right. \\
& \left.+\alpha_{2}^{u}+r_{2}^{u}\right) I_{2} \text {, } \\
& L V_{5}=\left(S+I_{1}+I_{2}\right)^{\theta}\left(A(t)-d(t) S-d(t) I_{1}-d(t) I_{2}\right. \\
& \left.-\alpha_{1}(t) I_{1}-\alpha_{2}(t) I_{2}\right)+\theta\left(S+I_{1}+I_{2}\right)^{\theta-1} \\
& \cdot\left(\frac{\sigma_{1}^{2}(t) S^{2} I_{1}^{2}}{\left(a_{1}(t)+I_{1}\right)^{2}}+\frac{\sigma_{2}^{2}(t) S^{2} I_{2}^{2}}{\left(a_{2}(t)+I_{2}\right)^{2}}\right) \leq A^{u}\left(S+I_{1}\right. \\
& \left.+I_{2}\right)^{\theta}-d^{l}\left(S+I_{1}+I_{2}\right)^{\theta+1}+\theta\left(S+I_{1}+I_{2}\right)^{\theta-1}\left(\sigma_{1}^{2 u}\right. \\
& \left.+\sigma_{2}^{2 u}\right) S^{2} \leq A^{u}\left(S+I_{1}+I_{2}\right)^{\theta}-\frac{1}{2}\left(d^{l}\right. \\
& \left.-\theta\left(\sigma_{1}^{2 u}+\sigma_{2}^{2 u}\right)\right)\left(S+I_{1}+I_{2}\right)^{\theta+1}-\frac{1}{2}\left(d^{l}\right. \\
& \left.-\theta\left(\sigma_{1}^{2 u}+\sigma_{2}^{2 u}\right)\right)\left(S+I_{1}+I_{2}\right)^{\theta+1} \leq O-\frac{1}{2}\left(d^{l}\right. \\
& \left.-\theta\left(\sigma_{1}^{2 u}+\sigma_{2}^{2 u}\right)\right) S^{\theta+1}-\frac{1}{2}\left(d^{l}-\theta\left(\sigma_{1}^{2 u}+\sigma_{2}^{2 u}\right)\right) I_{1}^{\theta+1} \\
& -\frac{1}{2}\left(d^{l}-\theta\left(\sigma_{1}^{2 u}+\sigma_{2}^{2 u}\right)\right) I_{2}^{\theta+1},
\end{aligned}
$$

$$
\begin{aligned}
& -\frac{1}{2}\left(d^{l}-\theta\left(\sigma_{1}^{2 u}+\sigma_{2}^{2 u}\right)\right) I_{1}^{\theta+1} \\
& -\frac{1}{2}\left(d^{l}-\theta\left(\sigma_{1}^{2 u}+\sigma_{2}^{2 u}\right)\right) I_{2}^{\theta+1}+O \\
& +d^{u}+\beta_{1}^{u}+\beta_{2}^{u}+\frac{\sigma_{1}^{2 u}}{2}+\frac{\sigma_{2}^{2 u}}{2} \\
= & -M \lambda-\frac{A^{l}}{S}+\left(d^{u}+\alpha_{1}^{u}+r_{1}^{u}\right) I_{1} \\
& +\left(d^{u}+\alpha_{2}^{u}+r_{2}^{u}\right) I_{2} \\
& -\frac{1}{2}\left(d^{l}-\theta\left(\sigma_{1}^{2 u}+\sigma_{2}^{2 u}\right)\right) S^{\theta+1} \\
& -\frac{1}{2}\left(d^{l}-\theta\left(\sigma_{1}^{2 u}+\sigma_{2}^{2 u}\right)\right) I_{1}^{\theta+1} \\
& -\frac{1}{2}\left(d^{l}-\theta\left(\sigma_{1}^{2 u}+\sigma_{2}^{2 u}\right)\right) I_{2}^{\theta+1}+O \\
& +d^{u}+\beta_{1}^{u}+\beta_{2}^{u}+\frac{\sigma_{1}^{2 u}}{2}+\frac{\sigma_{2}^{2 u}}{2} .
\end{aligned}
$$

Now, we are in the position to construct a compact subset $U$ such that $A_{2}$ in Lemma 2 holds. Define the following bounded closed set:

$$
\begin{aligned}
U & =\left\{\left(S, I_{1}, I_{2}\right) \in \mathbb{R}_{+}^{3}: \epsilon \leq S \leq \frac{1}{\epsilon}, \epsilon \leq I_{1} \leq \frac{1}{\epsilon}, \epsilon \leq I_{2}\right. \\
& \left.\leq \frac{1}{\epsilon}\right\},
\end{aligned}
$$

where $\epsilon>0$ is a sufficiently small number. In the set $\mathbb{R}_{+}^{3} \backslash U$, we can choose $\epsilon$ sufficiently small such that

$$
\begin{array}{r}
-M \lambda-\frac{A^{l}}{\epsilon}+C \leq-1, \\
-M \lambda+\left(d^{u}+\alpha_{1}^{u}+r_{1}^{u}\right) \epsilon+D \leq-1, \\
-M \lambda+\left(d^{u}+\alpha_{2}^{u}+r_{2}^{u}\right) \epsilon+E \leq-1, \\
-M \lambda-\frac{1}{2}\left(d^{l}-\theta\left(\sigma_{1}^{2 u}+\sigma_{2}^{2 u}\right)\right) \frac{1}{\epsilon^{\theta+1}}+C \leq-1, \\
-M \lambda-\frac{1}{4}\left(d^{l}-\theta\left(\sigma_{1}^{2 u}+\sigma_{2}^{2 u}\right)\right) \frac{1}{\epsilon^{\theta+1}}+G \leq-1, \\
-M \lambda-\frac{1}{4}\left(d^{l}-\theta\left(\sigma_{1}^{2 u}+\sigma_{2}^{2 u}\right)\right) \frac{1}{\epsilon^{\theta+1}}+H \leq-1,
\end{array}
$$

where $C, D, E, G$, and $H$ are positive constants which can be found from the following inequations (52), (54), (56), (59), and (61), respectively. For the sake of convenience, we divide into six domains, 


$$
\begin{aligned}
& U_{1}=\left\{\left(S, I_{1}, I_{2}\right) \in \mathbb{R}_{+}^{3}, 0<S<\epsilon\right\}, \\
& U_{2}=\left\{\left(S, I_{1}, I_{2}\right) \in \mathbb{R}_{+}^{3}, 0<I_{1}<\epsilon\right\}, \\
& U_{3}=\left\{\left(S, I_{1}, I_{2}\right) \in \mathbb{R}_{+}^{3}, 0<I_{2}<\epsilon\right\}, \\
& U_{4}=\left\{\left(S, I_{1}, I_{2}\right) \in \mathbb{R}_{+}^{3}, S>\frac{1}{\epsilon}\right\}, \\
& U_{5}=\left\{\left(S, I_{1}, I_{2}\right) \in \mathbb{R}_{+}^{3}, I_{1}>\frac{1}{\epsilon}\right\}, \\
& U_{6}=\left\{\left(S, I_{1}, I_{2}\right) \in \mathbb{R}_{+}^{3}, I_{2}>\frac{1}{\epsilon}\right\} .
\end{aligned}
$$

Next we will prove that $L \bar{V}\left(S, I_{1}, I_{2}\right) \leq-1$ on $\mathbb{R}_{+}^{3} \backslash U$, which is equivalent to proving it on the above six domains.

Case 1. If $\left(S, I_{1}, I_{2}\right) \in U_{1}$, one can see that

$$
\begin{aligned}
L \bar{V} \leq & -M \lambda-\frac{A^{l}}{S}+\left(d^{u}+\alpha_{1}^{u}+r_{1}^{u}\right) I_{1} \\
& +\left(d^{u}+\alpha_{2}^{u}+r_{2}^{u}\right) I_{2} \\
& -\frac{1}{2}\left(d^{l}-\theta\left(\sigma_{1}^{2 u}+\sigma_{2}^{2 u}\right)\right) I_{1}^{\theta+1} \\
& -\frac{1}{2}\left(d^{l}-\theta\left(\sigma_{1}^{2 u}+\sigma_{2}^{2 u}\right)\right) I_{2}^{\theta+1}+O+d^{u}+\beta_{1}^{u} \\
& +\beta_{2}^{u}+\frac{\sigma_{1}^{2 u}}{2}+\frac{\sigma_{2}^{2 u}}{2} \leq-M \lambda-\frac{A^{l}}{S}+C \\
\leq & -M \lambda-\frac{A^{l}}{\epsilon}+C,
\end{aligned}
$$

where

$$
\begin{aligned}
C & =\sup _{\left(S, I_{1}, I_{2}\right) \in R_{+}^{3}}\left\{\left(d^{u}+\alpha_{1}^{u}+r_{1}^{u}\right) I_{1}+\left(d^{u}+\alpha_{2}^{u}+r_{2}^{u}\right) I_{2}\right. \\
& -\frac{1}{2}\left(d^{l}-\theta\left(\sigma_{1}^{2 u}+\sigma_{2}^{2 u}\right)\right) I_{1}^{\theta+1} \\
& -\frac{1}{2}\left(d^{l}-\theta\left(\sigma_{1}^{2 u}+\sigma_{2}^{2 u}\right)\right) I_{2}^{\theta+1}+O+d^{u}+\beta_{1}^{u}+\beta_{2}^{u} \\
& \left.+\frac{\sigma_{1}^{2 u}}{2}+\frac{\sigma_{2}^{2 u}}{2}\right\} .
\end{aligned}
$$

According to (45), we have $L \bar{V} \leq-1$ for all $\left(S, I_{1}, I_{2}\right) \in U_{1}$.

Case 2. If $\left(S, I_{1}, I_{2}\right) \in U_{2}$, one can get that

$$
\begin{aligned}
L \bar{V} \leq & -M \lambda+\left(d^{u}+\alpha_{1}^{u}+r_{1}^{u}\right) I_{1}+\left(d^{u}+\alpha_{2}^{u}+r_{2}^{u}\right) I_{2} \\
& -\frac{1}{2}\left(d^{l}-\theta\left(\sigma_{1}^{2 u}+\sigma_{2}^{2 u}\right)\right) I_{2}^{\theta+1}+O+d^{u}+\beta_{1}^{u} \\
& +\beta_{2}^{u}+\frac{\sigma_{1}^{2 u}}{2}+\frac{\sigma_{2}^{2 u}}{2} \\
\leq & -M \lambda+\left(d^{u}+\alpha_{1}^{u}+r_{1}^{u}\right) I_{1}+D \\
\leq & -M \lambda+\left(d^{u}+\alpha_{1}^{u}+r_{1}^{u}\right) \epsilon+D,
\end{aligned}
$$

where

$$
\begin{aligned}
D & =\sup _{\left(S, I_{1}, I_{2}\right) \in R_{+}^{3}}\left\{\left(d^{u}+\alpha_{2}^{u}+r_{2}^{u}\right) I_{2}\right. \\
& -\frac{1}{2}\left(d^{l}-\theta\left(\sigma_{1}^{2 u}+\sigma_{2}^{2 u}\right)\right) I_{2}^{\theta+1}+O+d^{u}+\beta_{1}^{u}+\beta_{2}^{u} \\
& \left.+\frac{\sigma_{1}^{2 u}}{2}+\frac{\sigma_{2}^{2 u}}{2}\right\} .
\end{aligned}
$$

In view of (46), we can obtain that $L \bar{V} \leq-1$ for all $\left(S, I_{1}, I_{2}\right) \in U_{2}$.

Case 3. If $\left(S, I_{1}, I_{2}\right) \in U_{3}$, we have

$$
\begin{aligned}
L \bar{V} \leq & -M \lambda+\left(d^{u}+\alpha_{2}^{u}+r_{2}^{u}\right) I_{2}+\left(d^{u}+\alpha_{1}^{u}+r_{1}^{u}\right) I_{1} \\
& -\frac{1}{2}\left(d^{l}-\theta\left(\sigma_{1}^{2 u}+\sigma_{2}^{2 u}\right)\right) I_{1}^{\theta+1}+O+d^{u}+\beta_{1}^{u} \\
& +\beta_{2}^{u}+\frac{\sigma_{1}^{2 u}}{2}+\frac{\sigma_{2}^{2 u}}{2} \\
\leq & -M \lambda+\left(d^{u}+\alpha_{2}^{u}+r_{2}^{u}\right) I_{2}+E \\
\leq & -M \lambda+\left(d^{u}+\alpha_{2}^{u}+r_{2}^{u}\right) \epsilon+E,
\end{aligned}
$$

where

$$
\begin{aligned}
E & =\sup _{\left(S, I_{1}, I_{2}\right) \in R_{+}^{3}}\left\{\left(d^{u}+\alpha_{1}^{u}+r_{1}^{u}\right) I_{1}\right. \\
& -\frac{1}{2}\left(d^{l}-\theta\left(\sigma_{1}^{2 u}+\sigma_{2}^{2 u}\right)\right) I_{1}^{\theta+1}+O+d^{u}+\beta_{1}^{u}+\beta_{2}^{u} \\
& \left.+\frac{\sigma_{1}^{2 u}}{2}+\frac{\sigma_{2}^{2 u}}{2}\right\} .
\end{aligned}
$$
$U_{3}$.

By (47), we can conclude that $L \bar{V} \leq-1$ for all $\left(S, I_{1}, I_{2}\right) \in$

Case 4. If $\left(S, I_{1}, I_{2}\right) \in U_{4}$, one can derive that

$$
\begin{aligned}
L \bar{V} \leq & -M \lambda-\frac{1}{2}\left(d^{l}-\theta\left(\sigma_{1}^{2 u}+\sigma_{2}^{2 u}\right)\right) S^{\theta+1} \\
& +\left(d^{u}+\alpha_{1}^{u}+r_{1}^{u}\right) I_{1} \\
& -\frac{1}{2}\left(d^{l}-\theta\left(\sigma_{1}^{2 u}+\sigma_{2}^{2 u}\right)\right) I_{1}^{\theta+1} \\
& +\left(d^{u}+\alpha_{2}^{u}+r_{2}^{u}\right) I_{2} \\
& -\frac{1}{2}\left(d^{l}-\theta\left(\sigma_{1}^{2 u}+\sigma_{2}^{2 u}\right)\right) I_{2}^{\theta+1}+O+d^{u}+\beta_{1}^{u} \\
& +\beta_{2}^{u}+\frac{\sigma_{1}^{2 u}}{2}+\frac{\sigma_{2}^{2 u}}{2} \\
\leq & -M \lambda-\frac{1}{2}\left(d^{l}-\theta\left(\sigma_{1}^{2 u}+\sigma_{2}^{2 u}\right)\right) S^{\theta+1}+C \\
\leq & -M \lambda-\frac{1}{2}\left(d^{l}-\theta\left(\sigma_{1}^{2 u}+\sigma_{2}^{2 u}\right)\right) \frac{1}{\epsilon^{\theta+1}}+C .
\end{aligned}
$$


Together with (48), we can deduce that $L \bar{V} \leq-1$ for all $\left(S, I_{1}, I_{2}\right) \in U_{4}$.

Case 5. If $\left(S, I_{1}, I_{2}\right) \in U_{5}$, it follows that

$$
\begin{aligned}
L \bar{V} \leq & -M \lambda-\frac{1}{4}\left(d^{l}-\theta\left(\sigma_{1}^{2 u}+\sigma_{2}^{2 u}\right)\right) I_{1}^{\theta+1} \\
& +\left(d^{u}+\alpha_{1}^{u}+r_{1}^{u}\right) I_{1} \\
& -\frac{1}{4}\left(d^{l}-\theta\left(\sigma_{1}^{2 u}+\sigma_{2}^{2 u}\right)\right) I_{1}^{\theta+1} \\
& +\left(d^{u}+\alpha_{2}^{u}+r_{2}^{u}\right) I_{2} \\
& -\frac{1}{2}\left(d^{l}-\theta\left(\sigma_{1}^{2 u}+\sigma_{2}^{2 u}\right)\right) I_{2}^{\theta+1}+O+d^{u}+\beta_{1}^{u} \\
& +\beta_{2}^{u}+\frac{\sigma_{1}^{2 u}}{2}+\frac{\sigma_{2}^{2 u}}{2} \\
\leq & -M \lambda-\frac{1}{4}\left(d^{l}-\theta\left(\sigma_{1}^{2 u}+\sigma_{2}^{2 u}\right)\right) I_{1}^{\theta+1}+G \\
\leq & -M \lambda-\frac{1}{4}\left(d^{l}-\theta\left(\sigma_{1}^{2 u}+\sigma_{2}^{2 u}\right)\right) \frac{1}{\epsilon^{\theta+1}}+G,
\end{aligned}
$$

where

$$
\begin{aligned}
G & =\sup _{\left(S, I_{1}, I_{2}\right) \in R_{+}^{3}}\left\{\left(d^{u}+\alpha_{1}^{u}+r_{1}^{u}\right) I_{1}\right. \\
& -\frac{1}{4}\left(d^{l}-\theta\left(\sigma_{1}^{2 u}+\sigma_{2}^{2 u}\right)\right) I_{1}^{\theta+1}+\left(d^{u}+\alpha_{2}^{u}+r_{2}^{u}\right) I_{2} \\
& -\frac{1}{2}\left(d^{l}-\theta\left(\sigma_{1}^{2 u}+\sigma_{2}^{2 u}\right)\right) I_{2}^{\theta+1}+O+d^{u}+\beta_{1}^{u}+\beta_{2}^{u} \\
& \left.+\frac{\sigma_{1}^{2 u}}{2}+\frac{\sigma_{2}^{2 u}}{2}\right\} .
\end{aligned}
$$

By virtue of (49), we can deduce that $L \bar{V} \leq-1$ for all $\left(S, I_{1}, I_{2}\right) \in U_{5}$.

Case 6. If $\left(S, I_{1}, I_{2}\right) \in U_{6}$, we obtain

$$
\begin{aligned}
L \bar{V} \leq & -M \lambda-\frac{1}{4}\left(d^{l}-\theta\left(\sigma_{1}^{2 u}+\sigma_{2}^{2 u}\right)\right) I_{2}^{\theta+1} \\
& +\left(d^{u}+\alpha_{1}^{u}+r_{1}^{u}\right) I_{1} \\
& -\frac{1}{2}\left(d^{l}-\theta\left(\sigma_{1}^{2 u}+\sigma_{2}^{2 u}\right)\right) I_{1}^{\theta+1} \\
& +\left(d^{u}+\alpha_{2}^{u}+r_{2}^{u}\right) I_{2} \\
& -\frac{1}{4}\left(d^{l}-\theta\left(\sigma_{1}^{2 u}+\sigma_{2}^{2 u}\right)\right) I_{2}^{\theta+1}+O+d^{u}+\beta_{1}^{u} \\
& +\beta_{2}^{u}+\frac{\sigma_{1}^{2 u}}{2}+\frac{\sigma_{2}^{2 u}}{2} \\
\leq & -M \lambda-\frac{1}{4}\left(d^{l}-\theta\left(\sigma_{1}^{2 u}+\sigma_{2}^{2 u}\right)\right) I_{2}^{\theta+1}+H \\
\leq & -M \lambda-\frac{1}{4}\left(d^{l}-\theta\left(\sigma_{1}^{2 u}+\sigma_{2}^{2 u}\right)\right) \frac{1}{\epsilon^{\theta+1}}+H,
\end{aligned}
$$

where

$$
\begin{aligned}
H & =\sup _{\left(S, I_{1}, I_{2}\right) \in R_{+}^{3}}\left\{\left(d^{u}+\alpha_{1}^{u}+r_{1}^{u}\right) I_{1}\right. \\
& -\frac{1}{2}\left(d^{l}-\theta\left(\sigma_{1}^{2 u}+\sigma_{2}^{2 u}\right)\right) I_{1}^{\theta+1}+\left(d^{u}+\alpha_{2}^{u}+r_{2}^{u}\right) I_{2} \\
& -\frac{1}{4}\left(d^{l}-\theta\left(\sigma_{1}^{2 u}+\sigma_{2}^{2 u}\right)\right) I_{2}^{\theta+1}+O+d^{u}+\beta_{1}^{u}+\beta_{2}^{u} \\
& \left.+\frac{\sigma_{1}^{2 u}}{2}+\frac{\sigma_{2}^{2 u}}{2}\right\} .
\end{aligned}
$$

It follows from (50) that $L \bar{V} \leq-1$ for all $\left(S, I_{1}, I_{2}\right) \in U_{6}$.

Clearly, one can see from (52), (54), (56), (58), (59), and (61) that, for a sufficiently small $\epsilon$,

$$
\begin{aligned}
L \bar{V}\left(S, I_{1}, I_{2}\right) & \leq-1, \\
\left(S, I_{1}, I_{2}\right) & \in \mathbb{R}_{+}^{3} \backslash U .
\end{aligned}
$$

Hence $A_{2}$ in Lemma 2 is satisfied. This completes the proof of Theorem 5 .

\section{Extinction}

In this section, we investigate the conditions for the extinction of the two infectious diseases of system (3).

Let

$$
\begin{aligned}
\mathscr{R}_{1}= & \frac{A^{u}\left\langle\beta_{1}\right\rangle_{T}}{d^{l}\left\langle a_{1}\right\rangle_{T}\left\langle d+\alpha_{1}+r_{1}\right\rangle_{T}} \\
& -\frac{A^{2 u}\left\langle\sigma_{1}^{2}\right\rangle_{T}}{2 d^{2 l}\left\langle a_{1}^{2}\right\rangle_{T}\left\langle d+\alpha_{1}+r_{1}\right\rangle_{T}}, \\
\mathscr{R}_{2}= & \frac{A^{u}\left\langle\beta_{2}\right\rangle_{T}}{d^{l}\left\langle a_{2}\right\rangle_{T}\left\langle d+\alpha_{2}+r_{2}\right\rangle_{T}} \\
& -\frac{A^{2 u}\left\langle\sigma_{2}^{2}\right\rangle_{T}}{2 d^{2 l}\left\langle a_{2}^{2}\right\rangle_{T}\left\langle d+\alpha_{2}+r_{2}\right\rangle_{T}} .
\end{aligned}
$$

Theorem 6. Let $\left(S(t), I_{1}(t), I_{2}(t)\right)$ be a solution of system (3) with initial value $\left(S(0), I_{1}(0), I_{2}(0)\right) \in R_{+}^{3}$.

Then if

$$
\text { (i): }\left\langle\sigma_{i}^{2}\right\rangle_{T}>\frac{\left\langle\beta_{i}^{2}\right\rangle_{T}}{2\left\langle d^{l}+\alpha_{i}^{l}+r_{i}^{l}\right\rangle_{T}}, \quad i=1,2,
$$

or

$$
\text { (ii): } \mathscr{R}_{i}<1 \text {, }
$$

$$
\left\langle\sigma_{i}^{2}\right\rangle_{T} \leq \frac{d^{l}\left\langle a_{i}^{2}\right\rangle_{T}\left\langle\beta_{i}\right\rangle_{T}}{A^{u}\left\langle a_{i}\right\rangle_{T}},
$$

$$
i=1,2 \text {, }
$$

hold, the two infectious diseases of system (3) go to extinction a.s.; that is,

$$
\lim _{t \rightarrow+\infty} I_{i}(t)=0, \quad i=1,2 .
$$


Proof. Applying Itô's formula to system (3), we have

$$
\begin{array}{r}
\mathrm{d} \ln I_{i}(t)=\left(\frac{\beta_{i}(t) S(t)}{a_{i}(t)+I_{i}(t)}-\left(d(t)+\alpha_{i}(t)+r_{i}(t)\right)\right. \\
\left.-\frac{\sigma_{i}^{2}(t) S^{2}(t)}{2\left(a_{i}(t)+I_{i}(t)\right)^{2}}\right) \mathrm{d} t+\frac{\sigma_{i}(t) S(t)}{a_{i}(t)+I_{i}(t)} \mathrm{d} B_{i}(t), \\
\quad i=1,2 .
\end{array}
$$

Case (i). Integrating (68) from 0 to $t$ and dividing $t$ on both sides, we obtain

$$
\begin{aligned}
\frac{\ln I_{i}(t)}{t}= & -\frac{\sigma_{i}^{2}(t)}{2 t} \int_{0}^{t}\left(\frac{S(\tau)}{a_{i}(\tau)+I_{i}(\tau)}-\frac{\beta_{i}(\tau)}{\sigma_{i}^{2}(\tau)}\right)^{2} \mathrm{~d} \tau \\
& -\frac{1}{t} \int_{0}^{t}\left(d(\tau)+\alpha_{i}(\tau)+r_{i}(\tau)\right) \mathrm{d} \tau \\
& +\frac{1}{t} \int_{0}^{t} \frac{\beta_{i}^{2}(\tau)}{2 \sigma_{i}^{2}(\tau)} \mathrm{d} \tau+\frac{M_{i}(t)}{t}+\frac{\ln I_{i}(0)}{t} \\
\leq & -\left\langle d+\alpha_{i}+r_{i}-\frac{\beta_{i}^{2}}{2 \sigma_{i}^{2}}\right\rangle_{t}+\frac{M_{i}(t)}{t} \\
& +\frac{\ln I_{i}(0)}{t} .
\end{aligned}
$$

Case (ii). Integrating (68) from 0 to $t$ first and then dividing by $t$ on both sides yield

$$
\begin{aligned}
& \frac{\ln I_{i}(t)}{t}=\frac{1}{t} \int_{0}^{t}\left(\frac{\beta_{i}(\tau) S(\tau)}{a_{i}(\tau)+I_{i}(\tau)}\right. \\
& -\left(d(\tau)+\alpha_{i}(\tau)+r_{i}(\tau)\right) \\
& \left.\quad-\frac{\sigma_{i}^{2}(\tau) S^{2}(\tau)}{2\left(a_{i}(\tau)+I_{i}(\tau)\right)^{2}}\right) \mathrm{d} \tau+\frac{M_{i}(t)}{t}+\frac{\ln I_{i}(0)}{t} \\
& \leq\left\langle\frac{\beta_{i} A^{u}}{a_{i} d^{l}}-\left(d+\alpha_{i}+r_{i}\right)-\frac{\sigma_{i}^{2} A^{2 u}}{2 a_{i}^{2} d^{2 l}}\right\rangle_{t}+\frac{M_{i}(t)}{t} \\
& +\frac{\ln I_{i}(0)}{t} \leq\left\langle d+\alpha_{i}+r_{i}\right\rangle_{T}\left(\mathscr{R}_{i}-1\right)+\frac{M_{i}(t)}{t} \\
& +\frac{\ln I_{i}(0)}{t},
\end{aligned}
$$

where $M_{i}(t)=\int_{0}^{t} \sigma_{i}(\tau) S(\tau) /\left(a_{i}(\tau)+I_{i}(\tau)\right) \mathrm{d} B_{i}(\tau), i=1,2$, which is a local continuous martingale with $M_{i}(0)=0$. By Lemma 3, we have

$$
\lim _{t \rightarrow+\infty} \frac{M_{i}(t)}{t}=0, \quad i=1,2 .
$$

Taking the limit superior of both sides of (69) leads to

$$
\limsup _{t \rightarrow+\infty} \frac{\ln I_{i}(t)}{t} \leq-\left\langle d+\alpha_{i}+r_{i}-\frac{\beta_{i}^{2}}{2 \sigma_{i}^{2}}\right\rangle_{T}<0,
$$

which implies $\lim _{t \rightarrow+\infty} I_{i}(t)=0$.
Taking the superior limit of both sides of (70) leads to

$$
\limsup _{t \rightarrow+\infty} \frac{\ln I_{i}(t)}{t} \leq\left\langle d+\alpha_{i}+r_{i}\right\rangle_{T}\left(\mathscr{R}_{i}-1\right)<0,
$$

which implies $\lim _{t \rightarrow+\infty} I_{i}(t)=0, i=1,2$. This completes the proof.

Remark 7. Theorem 6 shows that the two diseases will die out if the white noise disturbance is large or the white noise disturbance is not large and $\mathscr{R}_{i}<1$. When $\left\langle\sigma_{i}^{2}\right\rangle_{T}>$ $\left\langle\beta_{i}^{2}\right\rangle_{T} / 2\left\langle d^{l}+\alpha_{i}^{l}+r_{i}^{l}\right\rangle_{T}$, the two infectious diseases of system (3) die out almost surely; that is to say, large white noise stochastic disturbance can lead to the two epidemics being extinct.

\section{Numerical Simulations}

Now we introduce some numerical simulations examples which illustrate our theoretical results.

Example 8. In model (3), let

$$
\begin{aligned}
& A(t)=0.5+0.1 \sin \pi t, \\
& a_{1}(t)=0.3+0.1 \sin \pi t, \\
& a_{2}(t)=0.31+0.1 \sin \pi t, \\
& \beta_{1}(t)=0.62+0.1 \sin \pi t, \\
& \beta_{2}(t)=0.62+0.1 \sin \pi t, \\
& r_{1}(t)=0.2+0.1 \sin \pi t, \\
& r_{2}(t)=0.35+0.1 \sin \pi t, \\
& d(t)=0.2+0.1 \sin \pi t, \\
& \alpha_{1}(t)=0.2+0.1 \sin \pi t, \\
& \alpha_{2}(t)=0.25+0.1 \sin \pi t, \\
& \sigma_{1}(t)=0.1+0.05 \sin \pi t, \\
& \sigma_{2}(t)=0.1+0.05 \sin \pi t .
\end{aligned}
$$

Note that $\mathfrak{R}>1, a_{1}(t) r_{1}(t) /\left(\beta_{1}(t)-a_{1}(t) d(t)\right)>A^{u} / d^{l}$, and $a_{2}(t) r_{2}(t) /\left(\beta_{2}(t)-a_{2}(t) d(t)\right)>A^{u} / d^{l}$ hold; that is, the conditions of Theorem 5 hold. Hence, system (3) has a positive periodic solution with $T=1$. Figure 1 (a) shows the periodicity of the nonautonomous stochastic model (3) with $\sigma_{1}=0$ and $\sigma_{2}=0$. Figure $1(\mathrm{~b})$ shows that solution of the nonautonomous stochastic model (3) with the initial value $\left(S(t), I_{1}(t), I_{2}(t)\right)=(0.3,0.15,0.15)$ tends to a periodic orbit in the sense of joint distribution.

Example 9. Choose the parameters in model (3) as follows:

$$
\begin{aligned}
& A(t)=0.2+0.2 \sin \pi t \\
& a_{1}(t)=0.23+0.2 \sin \pi t \\
& a_{2}(t)=0.25+0.2 \sin \pi t \\
& \beta_{1}(t)=0.2+0.2 \sin \pi t
\end{aligned}
$$




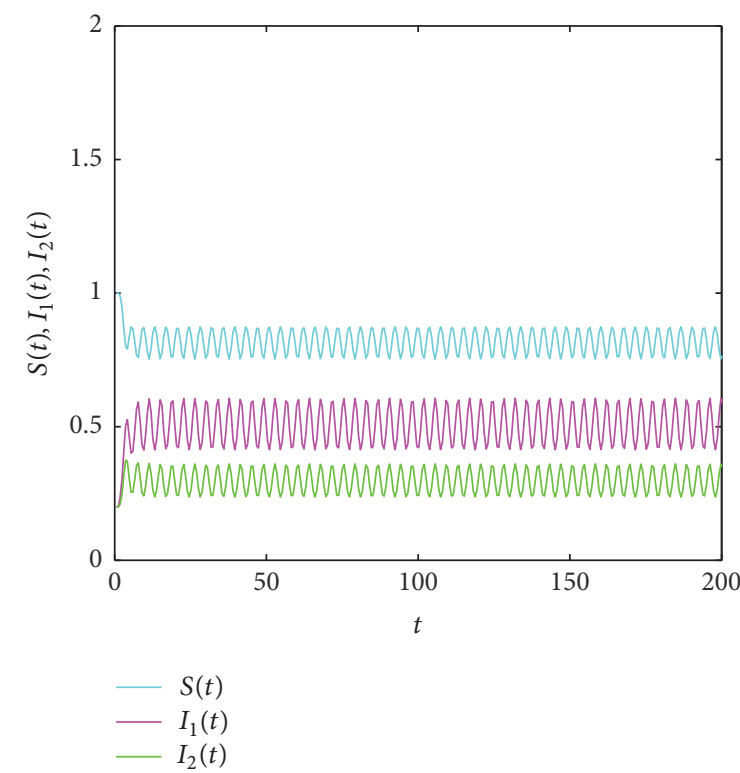

(a)

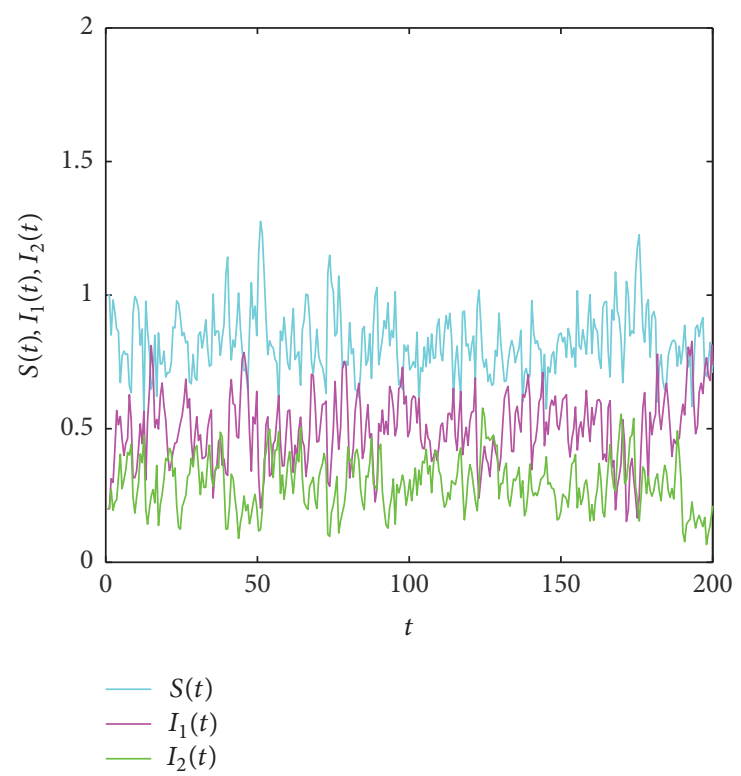

(b)

FIGURE 1: The solution $\left(S(t), I_{1}(t), I_{2}(t)\right)=(1,0.2,0.2)$ to the nonautonomous stochastic model $(3)$.
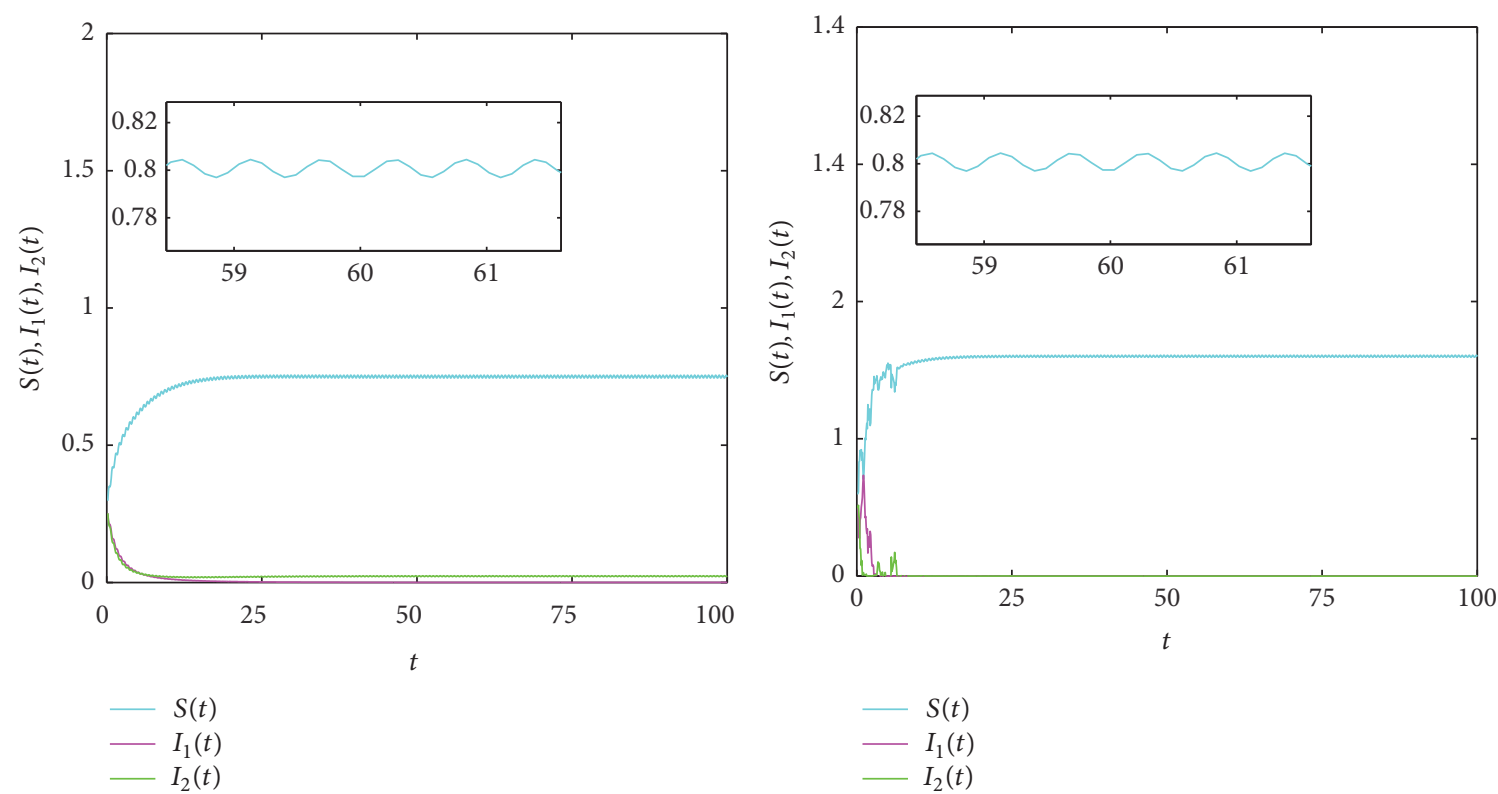

(a)

(b)

FIGURE 2: The solution $\left(S(t), I_{1}(t), I_{2}(t)\right)=(0.3,0.25,0.25)$ to the nonautonomous stochastic model (3).

$$
\begin{aligned}
\beta_{2}(t) & =0.3+0.2 \sin \pi t, \\
r_{1}(t) & =0.2+0.2 \sin \pi t, \\
r_{2}(t) & =0.3+0.2 \sin \pi t, \\
d(t) & =0.25+0.2 \sin \pi t, \\
\alpha_{1}(t) & =0.2+0.2 \sin \pi t, \\
\alpha_{2}(t) & =0.3+0.2 \sin \pi t, \\
\sigma_{1}(t) & =0.4+0.2 \sin \pi t, \\
\sigma_{2}(t) & =0.5+0.2 \sin \pi t .
\end{aligned}
$$

Note that $\left.\left\langle\sigma_{i}^{2}\right\rangle_{T}\right\rangle\left\langle\beta_{i}^{2}\right\rangle_{T} / 2\left\langle d^{l}+\alpha_{i}^{l}+r_{i}^{l}\right\rangle_{T}$. Therefore, conditions (i) of Theorem 6 hold. Then the two infectious diseases will go to extinction. Figure 2(a) shows that one of two diseases in the deterministic SIS epidemic model is extinct and the other is persistent. Figure 2(b) shows that the two diseases will die out under the large white noise disturbance of model (3).

Example 10. Choose the parameters in model (3) as follows:

$$
\begin{gathered}
A(t)=0.2+0.2 \sin \pi t, \\
a_{1}(t)=0.23+0.2 \sin \pi t,
\end{gathered}
$$




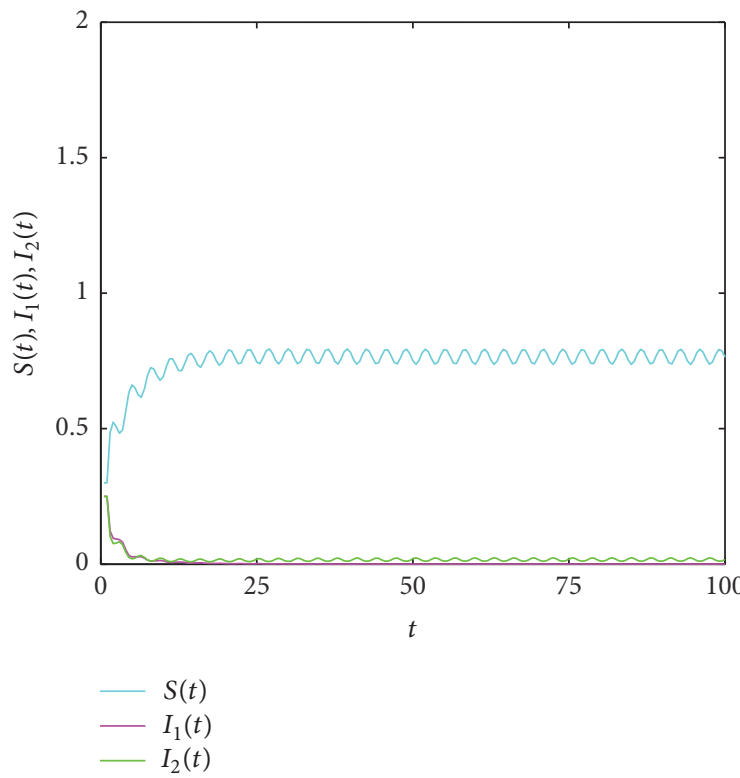

(a)

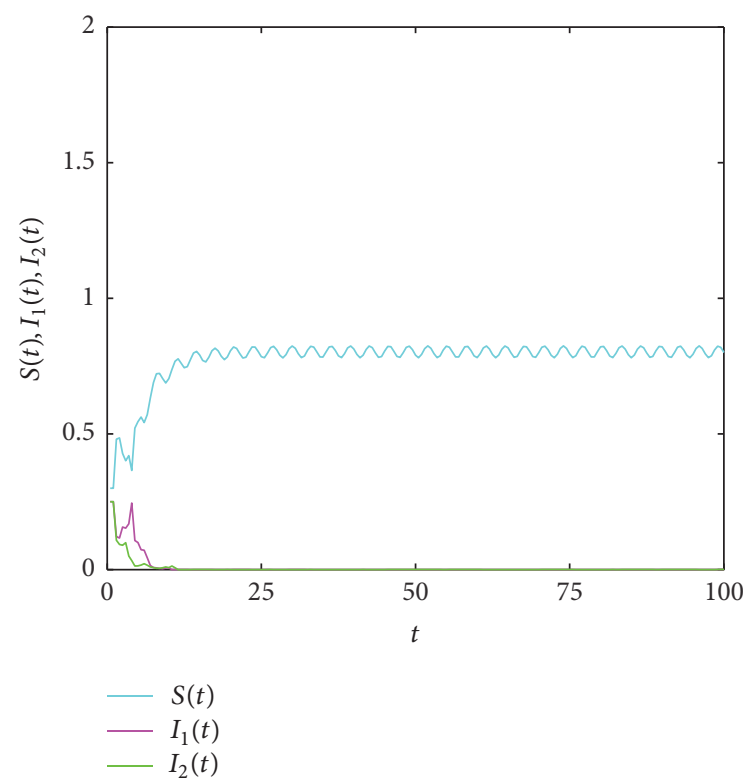

(b)

Figure 3: The solution $\left(S(t), I_{1}(t), I_{2}(t)\right)=(0.3,0.25,0.25)$ to the nonautonomous stochastic model (3).

$$
\begin{aligned}
& a_{2}(t)=0.25+0.2 \sin \pi t, \\
& \beta_{1}(t)=0.2+0.2 \sin \pi t, \\
& \beta_{2}(t)=0.3+0.2 \sin \pi t, \\
& r_{1}(t)=0.2+0.2 \sin \pi t, \\
& r_{2}(t)=0.3+0.2 \sin \pi t, \\
& d(t)=0.25+0.2 \sin \pi t, \\
& \alpha_{1}(t)=0.2+0.2 \sin \pi t, \\
& \alpha_{2}(t)=0.3+0.2 \sin \pi t, \\
& \sigma_{1}(t)=0.2+0.2 \sin \pi t, \\
& \sigma_{2}(t)=0.2+0.2 \sin \pi t .
\end{aligned}
$$

Note that $\mathscr{R}_{1}<1, \mathscr{R}_{2}<1$, and $\left\langle\sigma_{i}^{2}\right\rangle_{T} \leq d^{l}\left\langle a_{i}^{2}\right\rangle_{T}\left\langle\beta_{i}^{2}\right\rangle_{T} /$ $A^{u}\left\langle a_{i}\right\rangle_{T}$. That is, conditions (ii) of Theorem 6 hold. Then the two infectious diseases will go to extinction. Figure 3(a) shows that one of two diseases in the deterministic SIS epidemic model is extinct and the other is persistent without the white noises. Figure 3(b) shows that the two diseases will die out under a small white noise disturbance of model (3).

\section{Discussion and Conclusions}

This paper explores the existence of nontrivial positive T-periodic solution of a nonautonomous stochastic SIS epidemic model with nonlinear growth rate and double epidemic hypothesis. By constructing a suitable stochastic Lyapunov function, we establish sufficient conditions for the existence of nontrivial positive $T$-periodic solution of system (3). Furthermore, the sufficient conditions for the extinction of the two diseases are obtained. Our results are given as follows:

(1) If $a_{1}(t) r_{1}(t) /\left(\beta_{1}(t)-a_{1}(t) d(t)\right)>A^{u} / d^{l}, a_{2}(t) r_{2}(t) /$ $\left(\beta_{2}(t)-a_{2}(t) d(t)\right)>A^{u} / d^{l}$, and $R>1$ hold, the SIS model has at least one nontrivial positive $T$-periodic solution, where

$$
\mathfrak{R}=\sum_{i=1}^{2} \frac{\left\langle A \alpha_{i} \beta_{i}\right\rangle_{T}}{\left\langle d+\alpha_{i}+r_{i}+\sigma_{i}^{2} A^{2 u} / 2 a_{i}^{2} d^{2 l}\right\rangle_{T}\left\langle d+\sigma_{1}^{2} / 2+\sigma_{2}^{2} / 2\right\rangle_{T}\left\langle A+a_{1} \alpha_{1}+a_{2} \alpha_{2}\right\rangle_{T}} .
$$

(2) If $\left\langle\sigma_{i}^{2}\right\rangle_{T}>\left\langle\beta_{i}^{2}\right\rangle_{T} / 2\left\langle d^{l}+\alpha_{i}^{l}+r_{i}^{l}\right\rangle_{T}$, the two infectious diseases go extinct.

(3) If $\left\langle\sigma_{i}^{2}\right\rangle_{T} \leq \frac{d^{l}\left\langle a_{i}^{2}\right\rangle_{T}\left\langle\beta_{i}^{2}\right\rangle_{T}}{A^{u}\left\langle a_{i}\right\rangle_{T}}$ and $\mathscr{R}_{i}<1$, the two infectious diseases also go extinct, where

$$
\begin{aligned}
\mathscr{R}_{i}= & \frac{A^{u}\left\langle\beta_{i}\right\rangle_{T}}{d^{l}\left\langle a_{i}\right\rangle_{T}\left\langle d+\alpha_{i}+r_{i}\right\rangle_{T}} \\
& -\frac{A^{2 u}\left\langle\sigma_{i}^{2}\right\rangle_{T}}{2 d^{2 l}\left\langle a_{i}^{2}\right\rangle_{T}\left\langle d+\alpha_{i}+r_{i}\right\rangle_{T}} .
\end{aligned}
$$


Some interesting questions deserve further investigation. On the one hand, we may explore some realistic but complex models, such as considering the effects of impulsive or delay perturbations on system (3). On the other hand, we can concern the dynamics of a nonautonomous stochastic SIS epidemic model with two infectious diseases driven by Lévy jumps. What is more, we can also investigate the nonautonomous stochastic SIS epidemic model with two infectious diseases by a continuous time Markov chain. We will investigate these cases in our future work.

\section{Conflicts of Interest}

The authors declare that there are no conflicts of interest regarding the publication of this paper.

\section{Acknowledgments}

This work was supported by the National Natural Science Foundation of China (11371230), Joint Innovative Center for Safe and Effective Mining Technology and Equipment of Coal Resources, Shandong Province, the SDUST Research Fund (2014TDJH102), and Shandong Provincial Natural Science Foundation, China (ZR2015AQ001).

\section{References}

[1] W. O. Kermack and A. G. McKendrick, "A contributions to the mathematical theory of epidemics (Part I)," Proceedings of the Royal Society of London A, vol. 115, pp. 700-721, 1927.

[2] A. Gray, D. Greenhalgh, L. Hu, X. Mao, and J. Pan, "A stochastic differential equation SIS epidemic model," SIAM Journal on Applied Mathematics, vol. 71, no. 3, pp. 876-902, 2011.

[3] A. Korobeinikov and G. C. Wake, "Lyapunov functions and global stability for SIR, SIRS, and SIS epidemiological models," Applied Mathematics Letters, vol. 15, no. 8, pp. 955-960, 2002.

[4] H. W. Hethcote and P. van den Driessche, "An SIS epidemic model with variable population size and a delay," Journal of Mathematical Biology, vol. 34, no. 2, pp. 177-194, 1995.

[5] T. Zhang, X. Meng, Y. Song, and T. Zhang, "A stage-structured predator-prey SI model with disease in the prey and impulsive effects," Mathematical Modelling and Analysis, vol. 18, no. 4, pp. 505-528, 2013.

[6] J. Li and Z. Ma, "Qualitative analyses of SIS epidemic model with vaccination and varying total population size," Mathematical and Computer Modelling, vol. 35, no. 11-12, pp. 1235-1243, 2002.

[7] A. d'Onofrio, "A note on the global behaviour of the networkbased SIS epidemic model," Nonlinear Analysis: Real World Applications, vol. 9, no. 4, pp. 1567-1572, 2008.

[8] H. W. Hethcote and P. van den Driessche, "Two SIS epidemiologic models with delays," Journal of Mathematical Biology, vol. 40, no. 1, pp. 3-26, 2000.

[9] T. Zhang, X. Meng, T. Zhang, and Y. Song, "Global dynamics for a new high-dimensional SIR model with distributed delay," Applied Mathematics and Computation, vol. 218, no. 24, pp. 11806-11819, 2012.

[10] S. Gao, L. Chen, J. J. Nieto, and A. Torres, "Analysis of a delayed epidemic model with pulse vaccination and saturation incidence," Vaccine, vol. 24, no. 35-36, pp. 6037-6045, 2006.
[11] X. Meng, S. Zhao, T. Feng, and T. Zhang, "Dynamics of a novel nonlinear stochastic SIS epidemic model with double epidemic hypothesis," Journal of Mathematical Analysis and Applications, vol. 433, no. 1, pp. 227-242, 2016.

[12] A. Miao, X. Wang, T. Zhang, W. Wang, and B. Sampath Aruna Pradeep, "Dynamical analysis of a stochastic SIS epidemic model with nonlinear incidence rate and double epidemic hypothesis," Advances in Difference Equations, 2017:226 pages, 2017.

[13] X.-Z. Meng, "Stability of a novel stochastic epidemic model with double epidemic hypothesis," Applied Mathematics and Computation, vol. 217, no. 2, pp. 506-515, 2010.

[14] X. P. Li, X. Y. Lin, and Y. Q. Lin, "Lyapunov-type conditions and stochastic differential equations driven by G-Brownian motion," Journal of Mathematical Analysis and Applications, vol. 439, no. 1, pp. 235-255, 2016.

[15] M. Liu and M. Fan, "Permanence of stochastic Lotka-Volterra systems," Journal of Nonlinear Science, vol. 27, no. 2, pp. 425452, 2017.

[16] L. D. Liu and X. Z. Meng, "Optimal harvesting control and dynamics of two-species stochastic model with delays," Advances in Difference Equations, vol. 2017, 18 pages, 2017.

[17] H. Ma and Y. Jia, "Stability analysis for stochastic differential equations with infinite Markovian switchings," Journal of Mathematical Analysis and Applications, vol. 435, no. 1, pp. 593-605, 2016.

[18] G. D. Liu, X. H. Wang, X. Z. Meng, and S. J. Gao, "Extinction and persistence in mean of a novel delay impulsive stochastic infected predator-prey system with jumps," Complexity, vol. 3, pp. 1-15, 2017.

[19] C. Tan and W. H. Zhang, "On observability and detectability of continuous-time stochastic Markov jump systems," Journal of Systems Science and Complexity, vol. 28, no. 4, pp. 830-847, 2015.

[20] X. Leng, T. Feng, and X. Meng, "Stochastic inequalities and applications to dynamics analysis of a novel SIVS epidemic model with jumps," Journal of Inequalities and Applications, Paper No. 138, 25 pages, 2017.

[21] G. Li and M. Chen, "Infinite horizon linear quadratic optimal control for stochastic difference time-delay systems," Advances in Difference Equations, vol. 2015, 14 pages, 2015.

[22] Y. Zhao and W. Zhang, "Observer-based controller design for singular stochastic Markov jump systems with state dependent noise," Journal of Systems Science and Complexity, vol. 29, no. 4, pp. 946-958, 2016.

[23] S. Zhang, X. Meng, T. Feng, and T. Zhang, "Dynamics analysis and numerical simulations of a stochastic non-autonomous predator-prey system with impulsive effects," Nonlinear Analysis: Hybrid Systems, vol. 26, pp. 19-37, 2017.

[24] H.-j. Ma and T. Hou, "A separation theorem for stochastic singular linear quadratic control problem with partial information," Acta Mathematicae Applicatae Sinica, vol. 29, no. 2, pp. 303-314, 2013.

[25] X. Meng, L. Wang, and T. Zhang, "Global dynamics analysis of a nonlinear impulsive stochastic chemostat system in a polluted environment," Journal of Applied Analysis and Computation, vol. 6, no. 3, pp. 865-875, 2016.

[26] X. Liu, Y. Li, and W. Zhang, "Stochastic linear quadratic optimal control with constraint for discrete-time systems," Applied Mathematics and Computation, vol. 228, pp. 264-270, 2014. 
[27] X. Z. Meng and X. H. Wang, "Stochastic predator-prey system subject to lévy jump," Discrete Dynamics in Nature and Society, vol. 2016, Article ID 5749892, 13 pages, 2016.

[28] X. Lv, L. Wang, and X. Meng, "Global analysis of a new nonlinear stochastic differential competition system with impulsive effect," Advances in Difference Equations, vol. 2017, 296 pages, 2017.

[29] X. Zhang, D. Jiang, A. Alsaedi, and T. Hayat, "Stationary distribution of stochastic SIS epidemic model with vaccination under regime switching," Applied Mathematics Letters, vol. 59, pp. 87-93, 2016.

[30] F. Li, X. Meng, and Y. Cui, "Nonlinear stochastic analysis for a stochastic SIS epidemic model," Journal of Nonlinear Sciences and Applications, vol. 10, no. 09, pp. 5116-5124, 2017.

[31] A. Q. Miao, J. Zhang, T. Q. Zhang, and B. G. Pradeep, "Sampath Aruna Pradeep, Threshold dynamics of a stochastic SIR model with vertical transmission and vaccination," Computational and Mathematical Methods in Medicine, vol. 2017, Article ID 4820183, 10 pages, 2017.

[32] Q. Liu, D. Jiang, N. Shi, T. Hayat, and A. Alsaedi, "Nontrivial periodic solution of a stochastic non-autonomous SISV epidemic model," Physica A: Statistical Mechanics and its Applications, vol. 462, pp. 837-845, 2016.

[33] Y. L. Zhou, S. L. Yuan, and D. L. Zhao, "Threshold behavior of a stochastic SIS model with levy jumps," Applied Mathematics and Computation, vol. 275, pp. 255-267, 2016.

[34] T. Feng, X. Meng, L. Liu, and S. Gao, "Application of inequalities technique to dynamics analysis of a stochastic ecoepidemiology model," Journal of Inequalities and Applications, Paper No. 327, 29 pages, 2016.

[35] M. Liu and K. Wang, "Persistence and extinction in stochastic non-autonomous logistic systems," Journal of Mathematical Analysis and Applications, vol. 375, no. 2, pp. 443-457, 2011.

[36] Z. Bai and Y. Zhou, "Existence of two periodic solutions for a non-autonomous SIR epidemic model," Applied Mathematical Modelling: Simulation and Computation for Engineering and Environmental Systems, vol. 35, no. 1, pp. 382-391, 2011.

[37] S. Gao, F. Zhang, and Y. He, "The effects of migratory bird population in a nonautonomous eco-epidemiological model," Applied Mathematical Modelling: Simulation and Computation for Engineering and Environmental Systems, vol. 37, no. 6, pp. 3903-3916, 2013.

[38] T. Kuniya, "Existence of a nontrivial periodic solution in an agestructured SIR epidemic model with time periodic coefficients," Applied Mathematics Letters, vol. 27, pp. 15-20, 2014.

[39] L. Zu, D. Jiang, D. O'Regan, and B. Ge, "Periodic solution for a non-autonomous Lotka-Volterra predator-prey model with random perturbation," Journal of Mathematical Analysis and Applications, vol. 430, no. 1, pp. 428-437, 2015.

[40] Y. Lin, D. Jiang, and T. Liu, "Nontrivial periodic solution of a stochastic epidemic model with seasonal variation," Applied Mathematics Letters, vol. 45, pp. 103-107, 2015.

[41] X. R. Mao, Stochastic Differential Equations and Their Applications, Horwood, Chichester, UK, 1997.

[42] R. Khasminskii, Stochastic Stability of Differential Equations, Springer, Berlin, Germany, 2011. 


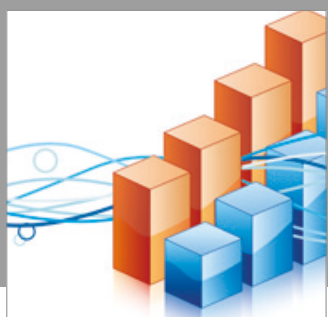

Advances in

Operations Research

vatersals

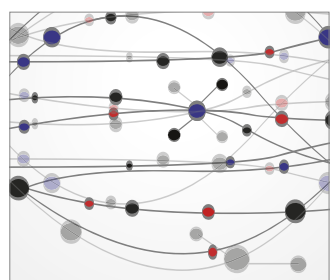

\section{The Scientific} World Journal
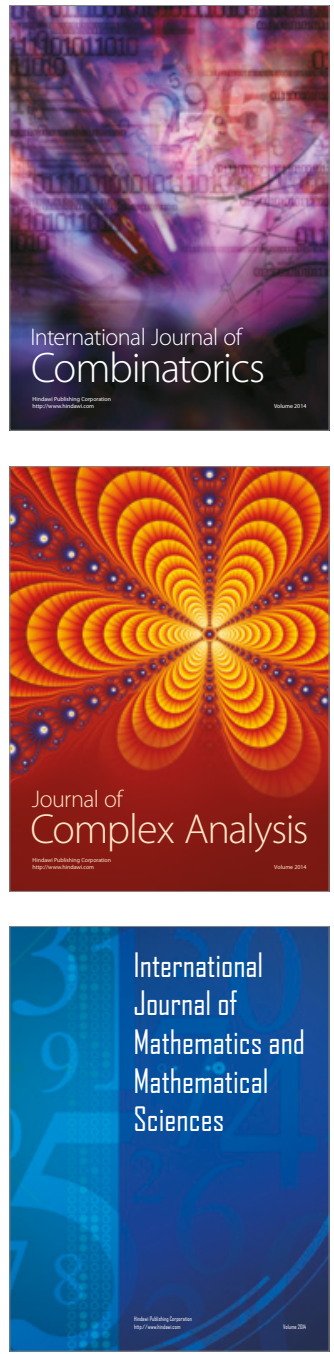
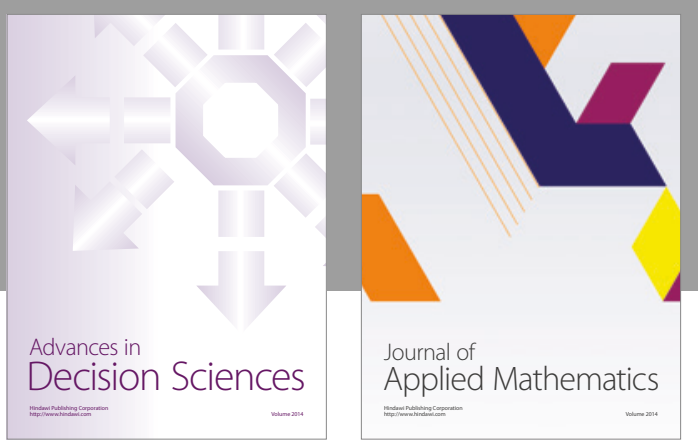

Algebra

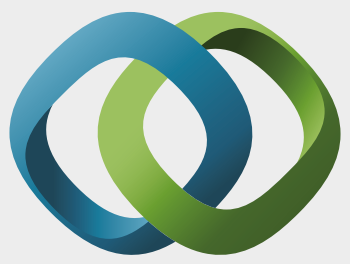

\section{Hindawi}

Submit your manuscripts at

https://www.hindawi.com
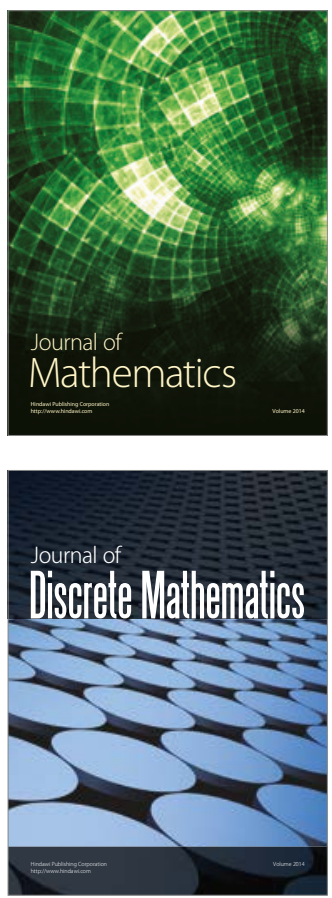

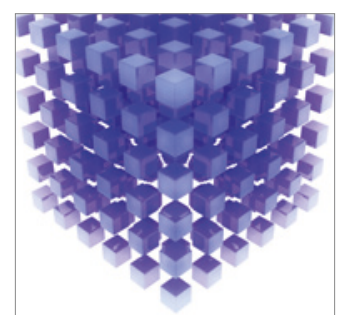

Mathematical Problems in Engineering
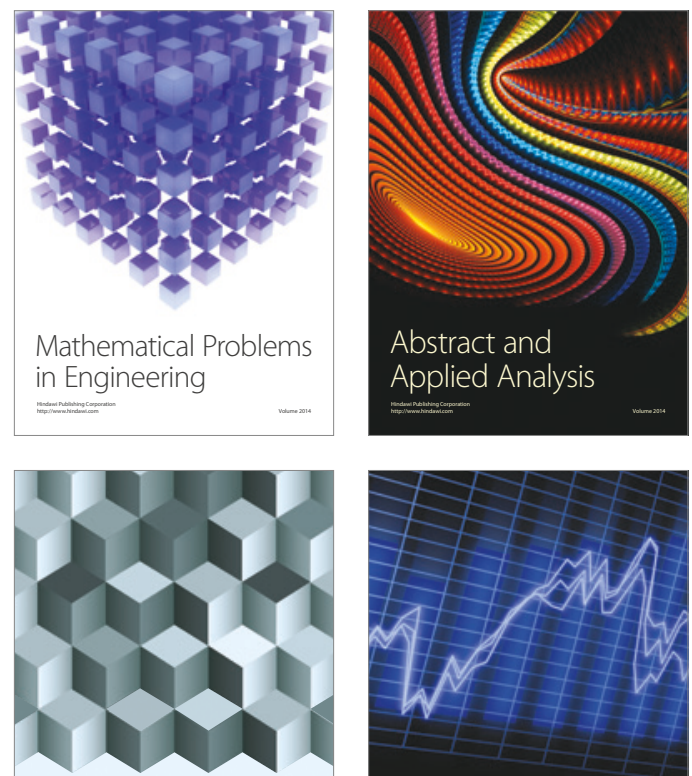

Journal of

Function Spaces

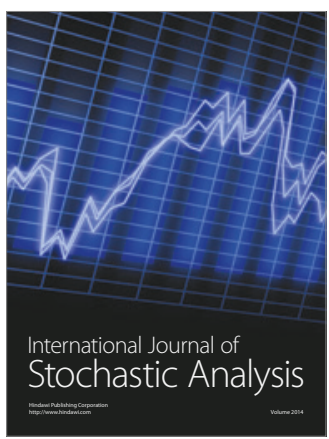

Probability and Statistics
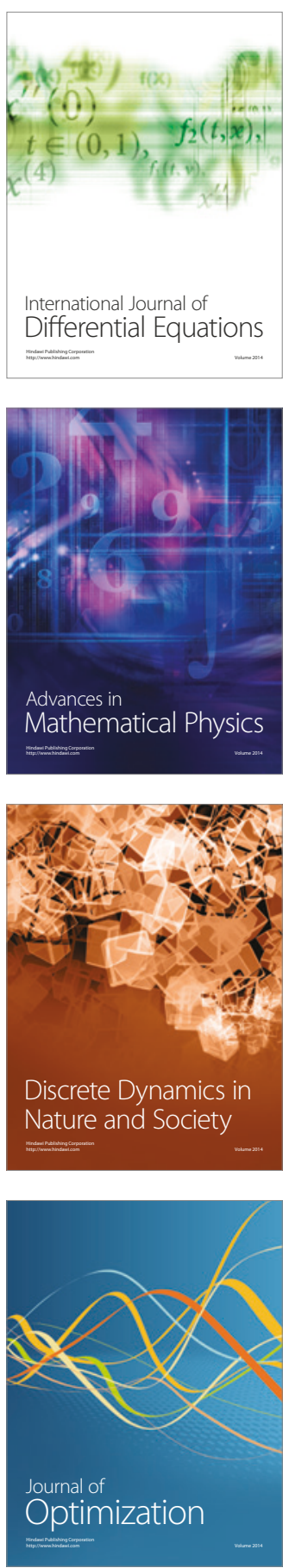